\title{
Shear instability and coherent structures in shallow flow adjacent to a porous layer
}

\author{
BRIAN L. WHITE ${ }^{1}$ AND HEIDI M. NEPF \\ ${ }^{1}$ Department of Physical Oceanography, Woods Hole Oceanographic Institution, \\ Woods Hole, MA, USA \\ ${ }^{2}$ Department of Civil and Environmental Engineering, Massachusetts Institute \\ of Technology, Cambridge, MA, USA
}

(Received 26 May 2006 and in revised form 5 July 2007)

Results are presented from an experimental study of shallow flow in a channel partially obstructed by an array of circular cylinders. The cylinder array is a model for emergent vegetation in an open channel, but also represents a simple sparse porous medium. A shear layer with regular vortex structures forms at the edge of the array, evolving downstream to an equilibrium width and vortex size. The vortices induce nearly periodic oscillations with a frequency that matches the most unstable linear mode for a parallel shear flow. The shear layer is asymmetric about the array interface and has a two-layer structure. An inner region of maximum shear near the interface contains a velocity inflection point and establishes the penetration of momentum into the array. An outer region, resembling a boundary layer, forms in the main channel, and establishes the scale of the vortices. The vortex structure, educed by conditional sampling, shows strong crossflows with sweeps from the main channel and ejections from the array, which create significant momentum and mass fluxes across the interface. The sweeps maintain the coherent structures by enhancing shear and energy production at the interface. A linear stability analysis is consistent with the experimental results and demonstrates that the instability is excited by the differential drag between the channel and the array.

\section{Introduction}

Shear flow at the interface between a porous layer and an open conduit is a problem of fundamental importance to a range of natural and engineered flows, e.g. laminar flows adjacent to foams, filters, or packed beds in industrial applications; turbulent flows at the sediment-fluid interface in rivers, estuaries and oceans; and flows adjacent to terrestrial or aquatic vegetation. The resistance within the porous layer reduces the velocity and creates a sharp transition across the interface. Of primary interest are the penetration of the velocity into the porous layer and the shear stress at the interface because these influence the discharge in the channel and the mass transport across the interface.

Whether laminar or turbulent, the flow within the porous layer is described by a bulk resistance law and flow in the outer layer is of open-channel or boundary-layer type. The transition between these layers must be determined. In a classic work, Beavers \& Joseph (1967) examined the laminar case, for which Darcy's law holds in the porous layer and Poiseuille flow governs the channel. The velocity at the porousfluid interface, $U_{s}$, termed the slip velocity, is related to the shear and the Darcy 
permeability, $k$, by

$$
\left.\frac{\partial\langle u\rangle}{\partial y}\right|_{y=0}=\frac{\beta}{\sqrt{k}} U_{s} .
$$

This condition gives a penetration length scale, $\sqrt{k} / \beta$, which is intrinsic to the porous medium, while the slip velocity depends on the applied shear, a function both of the porous medium and of the channel flow. However, experiments have shown the coefficient $\beta$ to vary substantially for porous media of different types and geometry.

Turbulent flow near a porous interface has been less frequently studied. However, there is a significant body of literature on turbulence above terrestrial forest and aquatic seagrass canopies (Raupach, Finnigan \& Brunet 1996; Finnigan 2000; Nepf \& Vivoni 2000). These canopies are typically sparser than the laminar porous layers discussed above, and have Reynolds numbers that are orders of magnitude larger. Nonetheless, as in the laminar case, penetration into the obstructed layer is set by a length scale associated with the resistance, in this case $\left(C_{D} a\right)^{-1}$, where the canopy density, $a$, is the area of solid obstructions projected to the flow per volume, and $C_{D}$ is the mean drag coefficient (Nepf et al. 2007; Poggi et al. 2004a,b).

Several studies in turbulent canopies have documented coherent vortices resulting from a Kelvin-Helmholtz instability initiated by the strong shear and velocity inflection at the top of the canopy (Raupach et al. 1996; Ghisalberti \& Nepf 2002). The coherent structures enhance scalar and momentum exchange, carrying highmomentum fluid into the canopy from above. Analogous horizontal vortices have been observed at the edge of a vegetated floodplain in a shallow channel (Tamai, Asaeda \& Ikeda 1986; Nezu \& Onitsuka 2000).

In addition to vegetated canopies, regular oscillations have been observed adjacent to a range of porous or roughness layers. Jimenez et al. (2001) found coherent vortices at the interface of a porous layer in numerical simulations, and demonstrated that they arise from a Kelvin-Helmholtz shear instability. Regular oscillations have also been observed in boundary-layer flows adjacent to permeable beds (Shvidchenko \& Pender 2001) and regular roughness arrays of, e.g. grooves (Ghaddar et al. 1986a; Djenidi, Elavarasan \& Antonia 1999) or spanwise cylinders (Schatz, Barkley \& Swinney 1995). Ghaddar et al. (1986a) found that the Kelvin-Helmholtz instability at the edge of the roughness layer excited coherent oscillations of the Tollmien-Schlichting channel mode, suggesting a link between shear-layer and boundary-layer instabilities. Ghaddar et al. (1986b), Djenidi et al. (1999) and Jimenez et al. (2001) have all noted that the coherent oscillations substantially increase the shear stress and scalar fluxes at the surface of these rough walls.

These results suggest that shear instability leading to coherent oscillations may be a common feature of flows adjacent to rough or porous layers at sufficiently large Reynolds number. In these flows, the roughness layer is permeable but imposes resistance, combining to create strong velocity shear and an inflection point, a recipe for shear instability according to Rayleigh's theorem for a inviscid parallel shear flow.

Here we describe experiments of moderate flows at high Reynolds number in a shallow channel partially filled with a cylinder array. The cylinder array, a simple porous layer, is a good model for aquatic macrophyte vegetation found in river floodplains, tidal marshes and freshwater wetlands. We observe regular periodic vortices at the edge of the array and advance a hypothesis to explain their dynamics. We first discuss the mean velocity and Reynolds-stress distributions measured in the experimental channel. We then describe a conditional sampling technique which allows us to educe the structure of the vortices. From this structure, we isolate the 

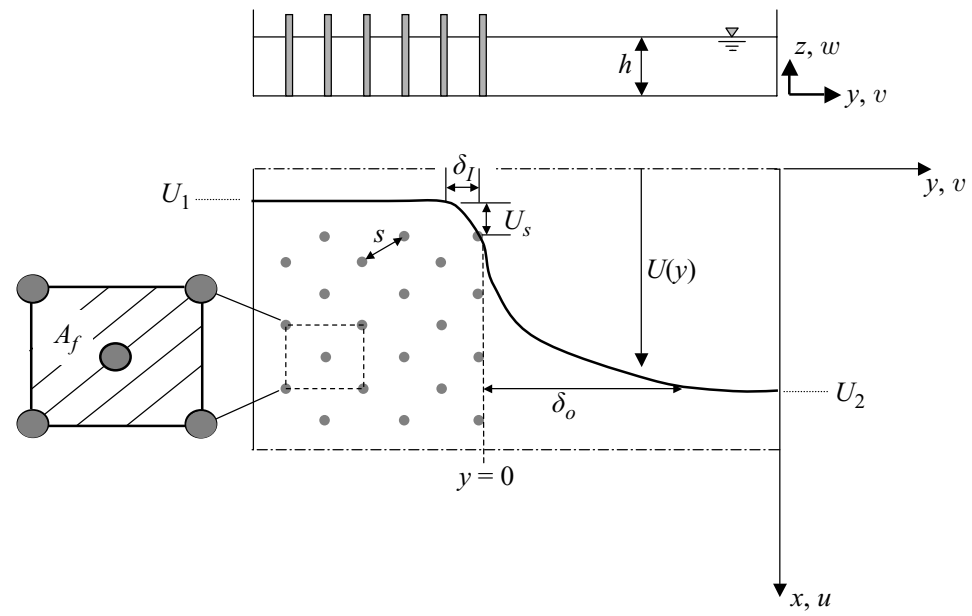

FIGURE 1. Problem description. Shallow laboratory channel with array of surface-piercing cylinders. Shown are the mean streamwise velocity, $U(y)$, the cylinder spacing, $s$, the slip velocity, $U_{s}$, the mean velocities in the vegetation and in the open channel, $U_{1}$ and $U_{2}$, and the inner- and outer-layer widths, $\delta_{I}$ and $\delta_{O} . A_{f}$ is the characteristic horizontal area over which the spatial average is taken.

process of momentum exchange and kinetic energy production, and hypothesize a cycle that sustains the vortices. Finally, we describe a linear stability analysis that demonstrates the effect of porous-layer resistance in promoting the shear instability.

\section{Problem description}

A shear flow is produced by partially filling a channel with an array of cylinders which extend through the water depth (figure 1). The flow is approximately twodimensional since the mean depth, $h$, is much smaller than the mean channel width, $B$. The cylinders are a simple morphological model for emergent marsh and riparian vegetation such as reeds and rushes, which exhibit very limited bending when exposed to currents (Leonard \& Luther 1995). The mean cylinder diameter is $d$, the solid volume fraction of the array is $\phi$, the porosity is $n=1-\phi$, and the average centre-tocentre distance between cylinders is $s$. The average solid frontal area per unit volume in the plane perpendicular to the flow is $a=N d$, where $N$ is the number density of cylinders (cylinders area ${ }^{-1}$ ). The interface, $y=0$, is taken as the outside tangent line along the outermost cylinder row.

The mean velocity is $U_{1}$ deep inside the array (defined as a time- and spatially averaged pore velocity) and increases, through a region of shear across the interface, to $U_{2}$ in the main channel. The overall velocity difference is $\Delta U=U_{2}-U_{1}$. Both $U_{1}$ and $U_{2}$ result from a balance between pressure gradient and frictional resistance, supplied in the array by cylinder drag, and in the channel by bottom friction. Two length scales characterize the velocity distribution: $\delta_{I}$, the length scale over which momentum penetrates into the array, and $\delta_{O}$, the length scale over which the shear extends into the main channel. These scales are termed the inner- and outerlayer widths, respectively, in analogy to rough boundary layers, and their physical characteristics are discussed in later sections. To describe the importance of viscous stresses, separate Reynolds numbers are defined for the array, $R e_{d}=\rho U_{1} d / \mu$, and for the open channel, $R e_{h}=\rho U_{2} h / \mu$, where $\mu$ is the dynamic viscosity of the fluid. 
Finally, the slip velocity at the interface, $U_{s}$, is defined as $U_{s}=U(y=0)-U_{1}$. For simplicity, in $\S 5.2, U_{s}$ will be defined at the inflection point of the velocity profile, which is always within a few diameters of the centreline.

\section{Momentum and resistance laws near a porous layer}

\subsection{Reynolds- and spatially averaged equations}

Here we present methods for averaging the equations of motion near a porous interface, and we highlight similarities between traditional porous media and vegetated flows. Consider two-dimensional flow in which half the plane is open and half is obstructed by a porous array (figure 1). Following Pedras \& de Lemos (2000), a fluid property, $\varphi$, a velocity component or pressure, is first time-averaged over a period, $\tau$, longer than pore scale turbulent fluctuations, then spatially averaged in the horizontal ( $x, y$-plane) over an area, $A_{f}$, a bed-parallel rectangle centred at $(x, y, z)$ with sides of length $s$ and including only interstitial fluid (see figure 1),

$$
\langle\bar{\varphi}\rangle(x, y, z, t)=\frac{1}{A_{f}} \int_{A_{f}}\left[\frac{1}{\tau} \int_{t}^{t+\tau} \varphi \mathrm{d} t\right] \mathrm{d} x \mathrm{~d} y .
$$

The overbar denotes the time average and the brackets the spatial average. Temporal and spatial fluctuations are

$$
\varphi^{\prime}=\varphi-\bar{\varphi} \quad \varphi^{\prime \prime}=\bar{\varphi}-\langle\bar{\varphi}\rangle .
$$

The average over the interstitial fluid, often called the intrinsic average, is related to the average taken over all space including the obstructions, called the superficial average, through the porosity, $\langle\bar{\varphi}\rangle^{s}=n\langle\bar{\varphi}\rangle$ (see, e.g. Breugem, Boersma \& Uittenbogaard 2006). Upon averaging the Navier-Stokes equations, the continuity and momentum equations are (using index notation)

$$
\begin{gathered}
\frac{\partial n\left\langle\bar{u}_{i}\right\rangle}{\partial x_{i}}=0, \\
\rho\left[n \frac{\partial\left\langle\bar{u}_{i}\right\rangle}{\partial t}+\frac{\partial n\left\langle\bar{u}_{j}\right\rangle\left\langle\bar{u}_{i}\right\rangle}{\partial x_{j}}\right]=-\frac{\partial n\langle\bar{p}\rangle}{\partial x_{i}}+\mu \frac{\partial^{2} n\left\langle\bar{u}_{i}\right\rangle}{\partial x_{j}^{2}}+\frac{\partial n\left\langle\tau_{i j}\right\rangle}{\partial x_{j}}+\rho n g-D_{i},
\end{gathered}
$$

where

$$
\left\langle\tau_{i j}\right\rangle=-\rho\left\langle\overline{u_{i}^{\prime} u_{j}^{\prime}}\right\rangle-\rho\left\langle\bar{u}_{i}^{\prime \prime} \bar{u}_{j}^{\prime \prime}\right\rangle
$$

is the macroscopic shear stress tensor, consisting of, respectively, turbulent Reynolds stresses, and dispersive stresses due to spatial fluctuations. The drag force, $D_{i}$, is the resistance due to the solid medium, the sum of form and viscous drag over the averaging scale,

$$
D_{i}=\left\langle\frac{\partial \bar{p}^{\prime \prime}}{\partial x_{i}}\right\rangle-\left\langle\mu \frac{\partial^{2} \bar{u}_{i}^{\prime \prime}}{\partial x_{j}^{2}}\right\rangle .
$$

Note that the spatial variation of the porosity, $n$, appears in (3.3). Even if the porosity is constant within the bulk of the medium, there is variation at the interface, for example, the averaging volume centred at $y=0$ has $n=n_{\text {bulk }} / 2$. However, in most vegetated flows, and in our experiments, $n \approx 1$, thus the spatial variation has little effect on the momentum balance. Presumably for this reason, it has been omitted from most canopy flow derivations (see, e.g. Raupach \& Thom 1981). The various resistance laws for flow in porous media can be derived from (3.3). When the array 
Reynolds number is small, $R e_{d} \rightarrow 0$, and there is no macro-scale shear, Darcy's law holds and the resistance is $D_{i}=\mu n^{2}\left\langle\bar{u}_{i}\right\rangle / k$, where $k$ is the permeability. Several approaches have been proposed for finite $R e$, most with a quadratic resistance law: the Forchheimer equation with $D_{i}=C \rho n^{2}\left\langle\bar{u}_{i}\right\rangle\left|\left\langle\bar{u}_{i}\right\rangle\right|$, the mixed Darcy-Forchheimer equation, $D_{i}=B \mu n^{2}\left\langle\bar{u}_{i}\right\rangle / k+C \rho n^{2}\left\langle\bar{u}_{i}\right\rangle\left|\left\langle\bar{u}_{i}\right\rangle\right|$, with $B$ and $C$ constants, and the DarcyForchheimer-Brinkman (DFB) equation when shear is included. In open channel or atmospheric vegetated flows the quadratic form,

$$
D_{i}=\frac{1}{2} \rho C_{D} a\left\langle\bar{u}_{i}\right\rangle\left|\left\langle\bar{u}_{i}\right\rangle\right|,
$$

is used, which is similar in form to the Forchheimer law. Koch \& Ladd (1997) have verified that a quadratic drag law describes the resistance at moderate-to-high Reynolds number in random cylinder arrays, much like those used in our experiments. We thus adopt the formulation (3.5) for the remainder of the paper.

\subsection{Depth-averaged flow}

We now turn to the shallow vegetated channel of our experimental conditions and demonstrate that it is a good approximation for two-dimensional flow near a porous layer. Because the flow is shallow, $h / B \ll 1$, where $B$ is the characteristic horizontal scale, the vertical motion is $O(h / B)$ compared with the horizontal, and the flow is predominantly two-dimensional. Nonetheless, the shear stress due to the bed, $\tau_{x z}=\mu \partial \bar{u} / \partial z-\rho \overline{u^{\prime} w^{\prime}}$ is the dominant momentum sink in the channel outside the vegetation. The three-dimensional equations of motion can be depth-averaged, yielding equations for the horizontal flow, while preserving the dynamic effect of the vertical shear as a uniform drag force. Defining time-, spatially- and depth-averaged quantities as

$$
\langle\bar{\varphi}\rangle_{d}=\frac{1}{h} \int_{0}^{h}\langle\bar{\varphi}\rangle \mathrm{d} z
$$

the two-dimensional shallow-water continuity and momentum equations, with $(i, j)=$ $(x, y)$, can be written

$$
\begin{gathered}
\frac{\partial n\left\langle\bar{u}_{i}\right\rangle_{d}}{\partial x_{i}}=0, \\
\rho n \frac{\partial\left\langle\bar{u}_{i}\right\rangle_{d}}{\partial t}+\rho \frac{\partial n\left\langle\bar{u}_{j}\right\rangle_{d}\left\langle\bar{u}_{i}\right\rangle_{d}}{\partial x_{j}}=-\rho g \frac{\partial n\langle\bar{h}\rangle_{d}}{\partial x_{i}}+\mu \frac{\partial^{2} n\left\langle\bar{u}_{i}\right\rangle_{d}}{\partial x_{j}^{2}}+\frac{\partial n\left\langle\tau_{i j}\right\rangle_{d}}{\partial x_{j}}-D_{i},
\end{gathered}
$$

where

$$
\left\langle\tau_{i j}\right\rangle_{d}=-\rho\left\langle\overline{u_{i}^{\prime} u_{j}^{\prime}}\right\rangle_{d}-\rho\left\langle\bar{u}_{i}^{\prime \prime} \bar{u}_{j}^{\prime \prime}\right\rangle_{d}-\rho\left\langle\left[\left\langle\bar{u}_{i}\right\rangle-\left\langle\bar{u}_{i}\right\rangle_{d}\right]\left[\left\langle\bar{u}_{j}\right\rangle-\left\langle\bar{u}_{j}\right\rangle_{d}\right]\right\rangle_{d}
$$

is the shear stress, as in (3.3), but the last term is a stress due to depth variations in the mean flow. The drag is due to the porous layer for $y \leqslant 0$, and the bed alone for $y>0$,

$$
D_{i}= \begin{cases}\frac{1}{2} \rho\left(C_{D} a+c_{f} / h\right)\left\langle\bar{u}_{i}\right\rangle_{d}\left|\left\langle\bar{u}_{i}\right\rangle_{d}\right|, & y<0, \\ \frac{1}{2} \rho\left(c_{f} / h\right)\left\langle\bar{u}_{i}\right\rangle_{d}\left|\left\langle\bar{u}_{i}\right\rangle_{d}\right|, & y>0,\end{cases}
$$

where $c_{f}$ is the bed friction coefficient, $\left|\left\langle\bar{u}_{i}\right\rangle_{d}\right|$ is the vector norm. The influence of bed drag will nearly always be negligible compared with drag from the obstructed layer (at most $\left(c_{f} / h\right) /\left(C_{D} a\right) \approx 0.01$ in our experiments). In writing the shallow-water equations in the form (3.7), the rigid-lid assumption has been made (for details, see Vreugdenhill 1994; Ghidaoui \& Kolyshkin 1999), which assumes variations in the 
depth, $h$, are small compared with the mean, except in the term representing the hydrostatic pressure gradient. This approximation is valid when the flow is subcritical and the surface gradient is small. These conditions are met in our experiments, where at most $\mathrm{d} h / \mathrm{d} x=O\left(1 \times 10^{-4}\right)$ and $F r=U / \sqrt{g h}<0.25$. Ghidaoui \& Kolyshkin (1999) showed that when $\mathrm{Fr}<1$, there is less than $5 \%$ difference between the growth rate of open-channel shear instability in the full shallow-water equations compared with the rigid-lid form. With the rigid-lid approximation, (3.7) are equivalent to the twodimensional Navier-Stokes equations in a closed conduit, with the surface slope acting as a pressure gradient, and the additional stress terms in (3.7c). As they satisfy the assumptions leading to the rigid-lid approximation, our experiments are comparable to two-dimensional flows in a closed conduit adjacent to a porous layer.

Equations (3.7) describe horizontal motions on time scales much longer than depthscale or vegetation-scale turbulence. Coherent unsteady motions on these time scales are well-documented in shallow free shear and wake flows (Uijttewaal \& Tukker 1998; Uijttewaal \& Booij 2000; Socolofsky \& Jirka 2004). Experiments have confirmed these flows are fundamentally two-dimensional, even exhibiting two-dimensional turbulence spectra (Uijttewaal \& Jirka 2003), primarily as a result of the large separation between the horizontal and depth scales. Similarly, the horizontal shear flow observed in our experiments, though dynamically influenced by bed friction, is predominantly twodimensional.

If a long-time average is taken to remove all temporal fluctuations, (3.7) reduce to

$$
0=-\rho g \frac{\mathrm{d} n\langle\bar{h}\rangle_{d}}{\mathrm{~d} x}+\mu \frac{\partial^{2} n U}{\partial y^{2}}+\frac{\partial n \tau_{x y}}{\partial y}-D_{x},
$$

where hereinafter $U$ denotes the mean depth-averaged velocity, the stress,

$$
\tau_{x y}=-\rho\left\langle\overline{u^{\prime} v^{\prime}}\right\rangle_{d}-\rho\left\langle\bar{u}^{\prime \prime} \bar{v}^{\prime \prime}\right\rangle_{d}-\rho\left\langle\left(\langle\bar{u}\rangle-\langle\bar{u}\rangle_{d}\right)\left(\langle\bar{v}\rangle-\langle\bar{v}\rangle_{d}\right)\right\rangle_{d},
$$

is composed of viscous, Reynolds, dispersive and secondary circulation contributions, and the drag is

$$
D_{x}= \begin{cases}\frac{1}{2} \rho\left(C_{D} a+c_{f} / h\right) U^{2}, & y<0, \\ \frac{1}{2} \rho\left(c_{f} / h\right) U^{2}, & y>0 .\end{cases}
$$

\subsection{Inner- and outer-layer scaling}

The shear flow across the porous-layer interface is characterized by two distinct length scales: the distance over which momentum penetrates the array; and the boundarylayer scale in the channel outside the array. Assuming the shear layer is unaffected by the channel width or the width of the array, the length scales in the problem are the water depth, $h$, the length scale associated with the array resistance, $\left(C_{D} a\right)^{-1}$ for high Reynolds number or $\sqrt{k}$ for Darcy resistance, and the cylinder diameter, $d$. The array penetration scale can be found by scaling the momentum equation in the sharp transition region across the interface, where the interfacial shear stress approximately balances the array resistance,

$$
\left.\left.\frac{\partial \tau_{x y}}{\partial y}\right|_{y=0} \sim D_{x}\right|_{y=0} .
$$


In the low $R e$ (Brinkman) limit, (3.9) reduces to a balance between viscous stress and Darcy resistance,

$$
\mu \frac{\partial^{2} U}{\partial y^{2}} \sim \mu \frac{U}{k} \Rightarrow \frac{\left.U\right|_{y=0}}{\delta_{I}^{2}} \sim \frac{\left.U\right|_{y=0}}{k},
$$

from which the inner-layer, or penetration, width is inversely related to the resistance

$$
\delta_{I} \sim \sqrt{k}
$$

Note that this result is also implied by the Beavers \& Joseph condition (1.1). For vegetated flows at higher $R e$, the dominant shear comes from the Reynolds stress, $\left\langle\overline{u^{\prime} v^{\prime}}\right\rangle$, since dispersive stresses are negligible for dense arrays, ad $\gtrsim 0.01$ (Poggi $2004 a, b$ ), and stress from secondary circulations is generally small (van Prooijen, Battjes \& Uijttewaal 2005). In this case, the depth-averaged momentum equation within the array (3.8) reduces to

$$
0=-g\langle\bar{h}\rangle_{d} \frac{\mathrm{d} n\langle\bar{h}\rangle_{d}}{\mathrm{~d} x}-\frac{\partial n\left\langle\overline{u^{\prime} v^{\prime}}\right\rangle_{d}}{\partial y}-\frac{1}{2}\left(C_{D} a\right) U^{2} .
$$

In analogy with boundary-layer flows, a friction velocity can be defined by the maximum stress at the interface,

$$
u_{*}^{2} \equiv-\left\langle\overline{u^{\prime} v^{\prime}}\right\rangle_{\max },
$$

which occurs very close to $y=0$. Then the balance near the interface between the shear stress and drag terms gives the scaling,

$$
\left.n \frac{u_{*}^{2}}{\delta_{I}} \sim C_{D} a U^{2}\right|_{y=0},
$$

which gives the scaling for the penetration width

$$
\delta_{I} \sim \frac{n u_{*}^{2}}{\left.U^{2}\right|_{y=0}}\left(C_{D} a\right)^{-1} .
$$

Both the laminar and high $R e$ scaling depend on a well-defined bulk resistance for the porous medium. Hence, $\delta_{I}$ must be larger than the scale of the representative averaging volume, $s$. When the penetration scale is smaller than the averaging scale, i.e. very dense media, local geometry prevails and $\delta_{I} \sim d$.

\subsection{Outer-layer scaling}

In the boundary layer outside the array, the shear stress approximately balances the pressure gradient from the free-surface slope. A scaling relationship for the outer-layer width, $\delta_{O}$, can be obtained by setting the pressure gradient and shear stress gradient from the momentum balance (3.8) to be of the same order (with $n=1$ in the channel),

$$
\frac{\partial\left\langle\overline{u^{\prime} v^{\prime}}\right\rangle}{\partial y} \sim g \frac{\mathrm{d}\langle\bar{h}\rangle_{d}}{\mathrm{~d} x} .
$$

From the uniform flow in the main channel outside the region of shear, we have

$$
g \frac{\mathrm{d}\langle\bar{h}\rangle_{d}}{\mathrm{~d} x}=-\frac{1}{2}\left(c_{f} / h\right) U_{2}^{2} .
$$



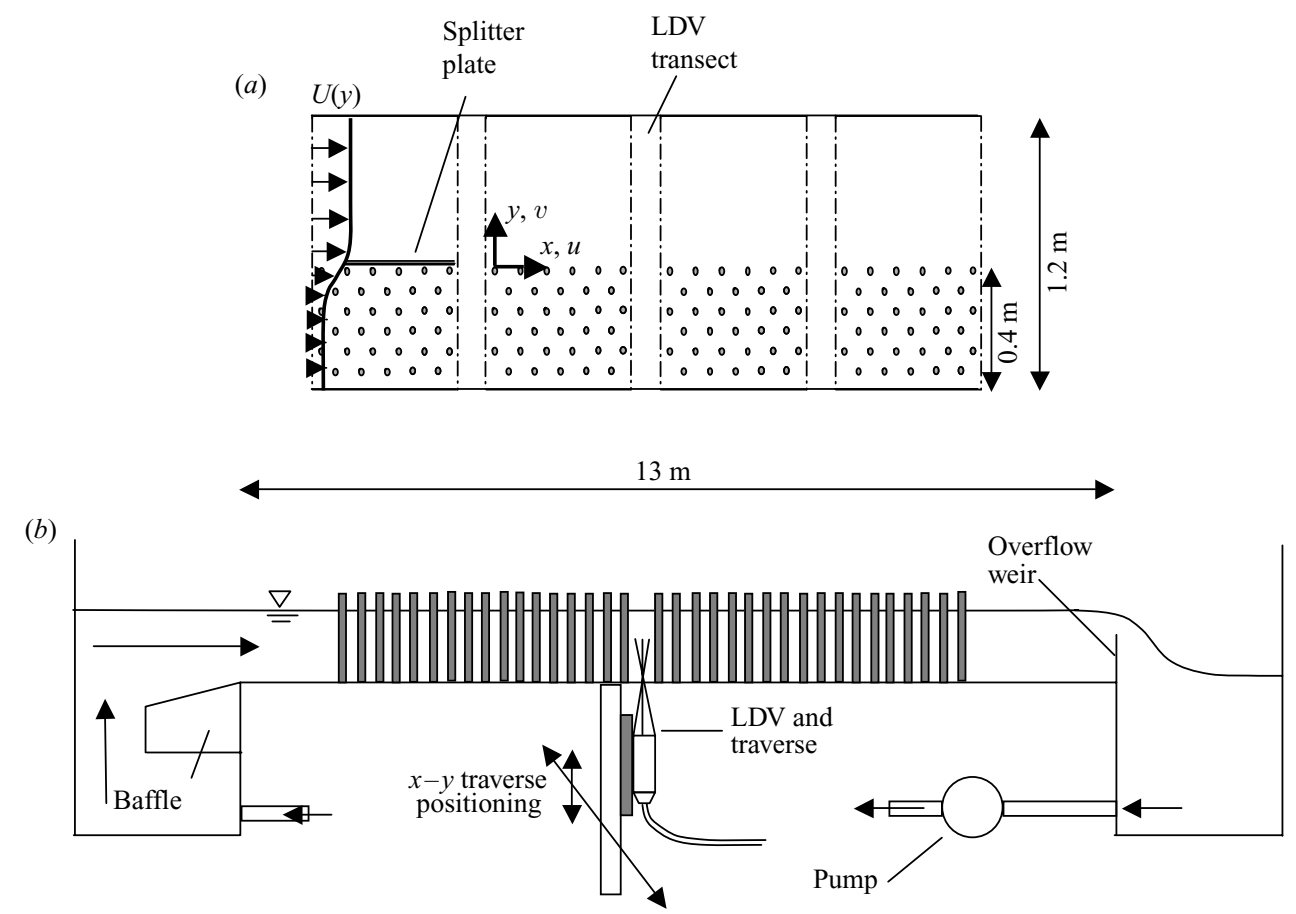

FIGURE 2. The laboratory set-up depicting the flume with LDV and traverse in profile $(b)$ and the cylinder array test section in plan view $(a)$.

Thus, substituting (3.17) into (3.16) gives the scaling

$$
\frac{u_{*}^{2}}{\delta_{O}} \sim \frac{1}{2}\left(c_{f} / h\right) U_{2}^{2},
$$

and the outer-layer scale is thus

$$
\delta_{O} \sim \frac{u_{*}^{2}}{U_{2}^{2}} \frac{2 h}{c_{f}} .
$$

Thus, the water depth in conjunction with the friction coefficient sets the channel boundary-layer scale. The scaling arguments for the outer layer (3.19) and inner layer (3.15) are confirmed by experimental data in $\S 5.2$.

\section{Experimental set-up}

To assess shear flow dynamics in the presence of a porous layer, experiments were carried out with model vegetation in a $1.2 \mathrm{~m}$ wide, $13 \mathrm{~m}$ long laboratory flume. A $40 \mathrm{~cm}$ wide array was created by placing $6 \mathrm{~mm}$ diameter wooden cylinders in a false bottom of perforated (1/4 in holes) PVC sheets (figure 2). The cylinders swelled when submerged, increasing the diameter to $6.5 \pm 0.2 \mathrm{~mm}$, and holding them rigidly in place. Solid volume fractions of $\phi=0.02, \phi=0.045$ and $\phi=0.10$ were studied. The cylinders were arranged in a staggered, equilateral array (see James \& Davis 2001). For this geometry, the solid fraction is related to the spacing and diameter by $\phi=(\sqrt{3} \pi / 6)\left(d^{2} / s^{2}\right)$.

As the flow entered the array, it was separated from the channel flow by a $1.2 \mathrm{~m}$ long, splitter plate, allowing the flow to develop separately within each region, and minimizing transverse motions due to flow adjustment (see Bousmar et al. 2005). 


\begin{tabular}{|c|c|c|c|c|c|c|c|c|c|c|c|}
\hline & I & II & III & IV & V & VI & VII & VIII & IX & $X$ & XI \\
\hline$\phi$ & 0.020 & 0.020 & 0.020 & 0.045 & 0.045 & 0.045 & 0.10 & 0.10 & 0.10 & 0.10 & 0.10 \\
\hline$C_{D} a \mathrm{~cm}^{-1}$ & 0.092 & 0.092 & 0.092 & 0.285 & 0.242 & 0.255 & 2.43 & 2.74 & 2.04 & 1.77 & 2.43 \\
\hline$s(\mathrm{~cm})$ & 4.4 & 4.4 & 4.4 & 2.9 & 2.9 & 2.9 & 2.0 & 2.0 & 2.0 & 2.0 & 2.0 \\
\hline$h(\mathrm{~cm})$ & 6.8 & 10.4 & 13.8 & 6.6 & 5.3 & 6.0 & 6.6 & 5.5 & 6.8 & 7.8 & 13.9 \\
\hline $\operatorname{Re}_{\theta}\left(\times 10^{3}\right)$ & 8.2 & 12.0 & 14.0 & 7.6 & 1.6 & 6.0 & 6.9 & 2.4 & 3.6 & 12.0 & 11.0 \\
\hline $\operatorname{Re}_{h}\left(\times 10^{3}\right)$ & 11.0 & 21.0 & 30.0 & 10.0 & 1.8 & 6.7 & 10.0 & 2.9 & 5.6 & 21.0 & 28.0 \\
\hline$U_{1}\left(\mathrm{~cm} \mathrm{~s}^{-1}\right)$ & 2.2 & 1.7 & 1.9 & 1.3 & 0.3 & 0.8 & 0.4 & 0.2 & 0.3 & 0.9 & 0.4 \\
\hline$U_{2}\left(\mathrm{~cm} \mathrm{~s}^{-1}\right)$ & 17.7 & 21.7 & 24.0 & 17.4 & 3.8 & 12.3 & 16.8 & 5.9 & 9.1 & 29.6 & 22.0 \\
\hline$u_{*}\left(\mathrm{~cm} \mathrm{~s}^{-1}\right)$ & 1.8 & 2.3 & 2.7 & 2.1 & 0.4 & 1.5 & 1.9 & 0.4 & 0.8 & 3.4 & 2.5 \\
\hline$U_{s}\left(\mathrm{~cm} \mathrm{~s}^{-1}\right)$ & 3.7 & 5.1 & 5.6 & 3.7 & 0.8 & 2.5 & 3.4 & 1.0 & 1.8 & 6.1 & 4.5 \\
\hline$y_{o}$ & 1.0 & 1.6 & 1.1 & -1.0 & -0.6 & -1.0 & 0.2 & 0.5 & 0.2 & 0.0 & 0.5 \\
\hline$\delta_{I}(\mathrm{~cm})$ & 3.7 & 6.0 & 6.2 & 2.6 & 2.2 & 1.9 & 1.2 & 0.9 & 1.1 & 1.3 & 1.4 \\
\hline$\delta_{O}(\mathrm{~cm})$ & 16.0 & 19.1 & 19.9 & 16.7 & 16.9 & 18.2 & 16.5 & 15.5 & 15.2 & 17.8 & 21.5 \\
\hline$U_{m}\left(\mathrm{~cm} \mathrm{~s}^{-1}\right)$ & 7.4 & 10.2 & 12.0 & 7.9 & 1.6 & 5.6 & 6.7 & 2.1 & 3.6 & 12.0 & 9.0 \\
\hline$y_{m}(\mathrm{~cm})$ & 3.8 & 4.8 & 4.3 & 1.2 & 1.4 & 0.8 & 1.9 & 1.8 & 1.7 & 1.9 & 2.5 \\
\hline$\theta_{e q}(\mathrm{~cm})$ & 5.1 & 6.3 & 6.2 & 4.8 & 4.5 & 5.4 & 4.5 & 4.4 & 4.4 & 4.4 & 5.5 \\
\hline
\end{tabular}

TABLE 1. Experimental parameters and results for each case (from equilibrium profiles).

The flow depth, $h$, was varied between $5.5 \mathrm{~cm}$ and $15 \mathrm{~cm}$, maintaining shallow flow conditions with depth to width ratios of $h / B \ll 1$. The channel and the cylinder array were wide enough to ensure the shear layer for all cases was unaffected by the flume sidewalls. A recirculating pump provided flows in the range $2-501 \mathrm{~s}^{-1}$ and Reynolds numbers of $O\left(10^{3}-10^{4}\right)$ based on flow depth, $R e_{h}=\rho U_{2} h / \mu$. Experimental configurations are summarized in table 1.

Free-surface elevation was measured using analogue capacitance-based displacement gauges connected to an A/D board and sampled at $25 \mathrm{~Hz}$. Elevation at the upstream and downstream ends of the cylinder array were used to measure surface slope, $\mathrm{d}\langle\bar{h}\rangle / \mathrm{d} x$, from which the drag coefficient of the array was estimated. The difference in surface elevation relative to flow depth was $O\left(10^{-2}\right)$ or smaller over the length of the flume, thus the effect of depth variation on streamwise velocity was negligible.

Simultaneous two-component velocity measurements were taken in the horizontal plane with a laser-doppler velocimetry (LDV) system in backscatter mode (Dantec Dynamics). The LDV was mounted on a positioning system driven by a stepper motor, with resolution better than $0.1 \mathrm{~mm}$. Lateral velocity transects were made at various longitudinal positions downstream of the splitter plate. To ensure a clear optical path for the LDV beams, 0.5 in wide, cast acrylic spacers were placed between adjacent PVC base boards.

Most measurements were made at mid-depth, as a proxy for the depth-averaged velocity. In the channel outside the cylinder array, vertical profiles were logarithmic. In the array, the profiles were nearly uniform over depth. In both regions, the mid-depth measurements were found to be within $5 \%$ of the true depth-averaged velocity.

\section{Experimental results}

\subsection{Development of the mean flow}

Lateral profiles of streamwise velocity are shown with increasing distance from the splitter plate in figure 3(a). A shear layer develops, with an inflection point near the interface, as the slow and fast streams mix. Initially, like a classic mixing layer (Brown \& Roshko 1974; Browand \& Weidman 1976; Ho \& Huerre 1984), the shear in 
(a)

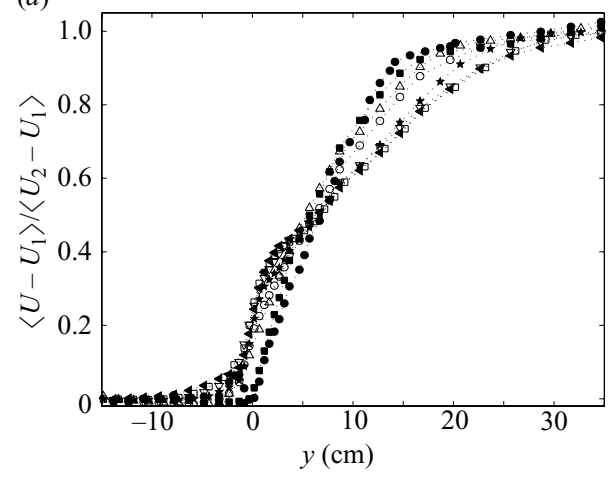

(c)

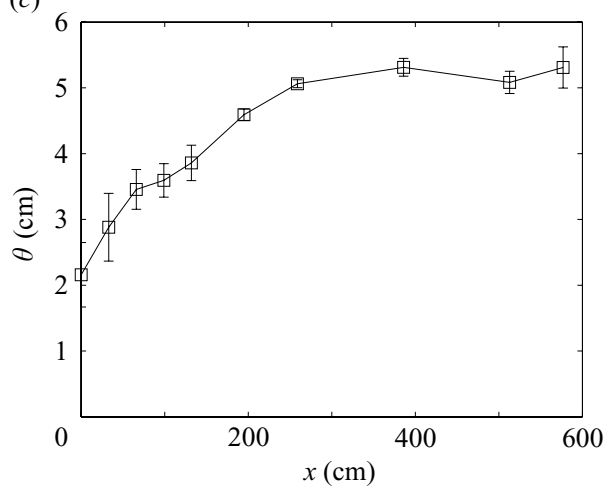

(b)

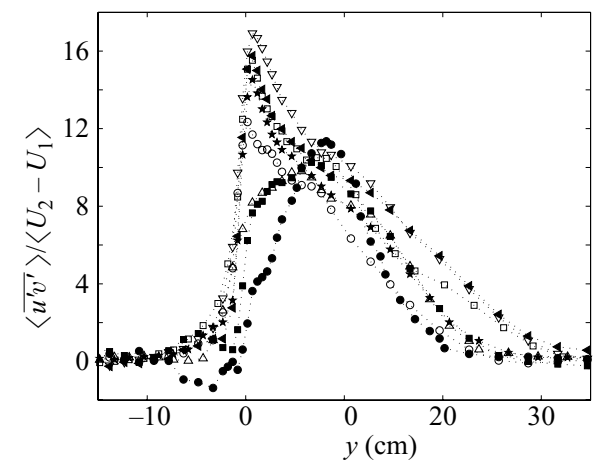

FIGURE 3. Experimental LDV measurements of shear-layer growth downstream of the splitter plate (case IV). (a) Streamwise velocity, (b) Reynolds stress, $(c)$ momentum thickness. Position markers are $x=0(\bullet), x=33 \mathrm{~cm}(\mathbf{\square}), x=66 \mathrm{~cm}(\triangle), x=132 \mathrm{~cm}(\bigcirc), x=195 \mathrm{~cm}(\star), x=386 \mathrm{~cm}$ $(\square), x=513 \mathrm{~cm}(\nabla), x=577 \mathrm{~cm}(\varangle)$. Only the region of shear is shown $(-15 \mathrm{~cm}<y<35 \mathrm{~cm})$, whereas actual flume dimensions are $-40 \mathrm{~cm}<y<80 \mathrm{~cm}$.

the outer region decreases while the shear layer width grows, indicating a net flux of momentum toward the array. However, the array drag limits momentum penetration. As the maximum penetration is established $(x \approx 400 \mathrm{~cm}$, from figure $3 a)$ a kink develops in the velocity profile near the interface. The shear-layer width, measured by the momentum thickness,

$$
\theta=\int_{-\infty}^{\infty}\left[\frac{1}{4}-\left(\frac{U-\bar{U}}{\Delta U}\right)^{2}\right] \mathrm{d} y,
$$

initially grows, but asymptotes to approximately $\theta=5 \mathrm{~cm}$ by $x \approx 400 \mathrm{~cm}$ (figure $3 c$ ). Here $\bar{U}=\left(U_{1}+U_{2}\right) / 2$. The final three profiles in figure 3(a) line up exactly, suggesting an equilibrium by $x \approx 400 \mathrm{~cm}$, in agreement with the asymptotic behavior of $\theta$. The equilibrium width is denoted $\theta_{e q}$.

The development of the Reynolds stress distribution is shown in figure 3(b). As the flow develops, the peak shifts toward the interface and becomes more pronounced. The peak in the equilibrium Reynolds stress profile coincides with the velocity inflection point, which is within $1-2 \mathrm{~cm}$ of the array edge. The coincidence of the Reynolds stress maximum and the inflection point was observed for all experimental cases. Thus, we 
(a)

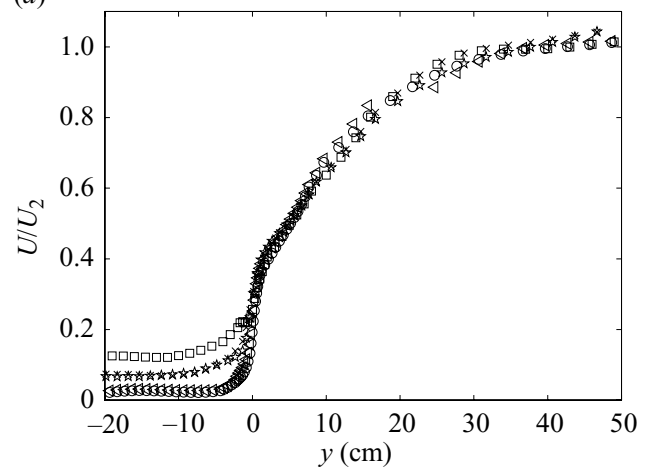

(b)

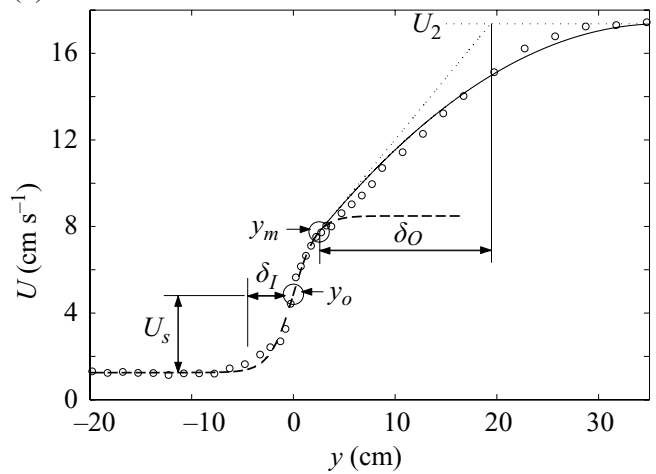

Figure 4. (a) Equilibrium velocity profiles: case I, $\phi=0.02(\square)$; case IV, $\phi=0.045(\times)$; case VI, $\phi=0.045(\star)$; case VII, $\phi=0.1(\bigcirc)$; case X, $\phi=0.1(\triangleleft)$. (b) Representative profile demonstrating the inner layer $\left(\delta_{I}\right)$, outer layer $\left(\delta_{O}\right)$, and associated variables.

define an interfacial shear stress, $\tau_{i}$, and friction velocity, $u_{*}$, at the maximum,

$$
\tau_{i}=u_{*}^{2}=-\left\langle\overline{u^{\prime} v^{\prime}}\right\rangle_{\max } .
$$

Because the maximum shear and maximum Reynolds stress coincide there, the interface is a point of high energy production, $P=-\left\langle\overline{u^{\prime} v^{\prime}}\right\rangle \partial u / \partial y$.

\subsection{Two-layer scaling of the equilibrium velocity}

Equilibrium mean velocity profiles for three cylinder volume fractions, $\phi$, are shown in figure $4(a)$. The velocity was spatially averaged with a moving filter of window length $s$ to remove cylinder-scale spatial fluctuations in the array. In the region near the interface, both the degree of penetration and the magnitude of the pore velocity, $U_{1}$, decrease with increasing $\phi$. However, in the outer region, the normalized velocity, $U / U_{2}$, is nearly independent of density, suggesting the array has little influence on the structure of the outer flow. This confirms the scaling analysis in $\$ \S 3.3$ and 3.4 , which predicted a distinct inner- and outer-layer structure for the velocity distribution, with independent length scales $\delta_{I}$ and $\delta_{O}$. These scales are labelled in figure $4(b)$.

Because of the two-layer structure, different scaling laws apply to the velocity profile in the inner and outer layers. The velocity inflection point at the interface suggests the inner layer can be scaled as a shear layer, while the broad outer region suggests a boundary-layer scaling. In the inner layer, a rescaled velocity, $\left(U-U_{1}\right) /\left(2 U_{s}\right)$, can be plotted against an inner-layer coordinate, $\left(y-y_{o}\right) / \delta_{I}$. The reference $y_{o}$ is the shearlayer inflection point, $U_{s}=U\left(y_{o}\right)-U_{1}$ is the slip velocity, and $\delta_{I}$ is the inner layer width. We measure $\delta_{I}, y_{o}$ and $U_{s}$ for each measured velocity profile by a nonlinear regression to a hyperbolic tangent profile,

$$
u=U_{1}+U_{s}\left(1+\tanh \left(\frac{y-y_{o}}{\delta_{I}}\right)\right) .
$$

The hyperbolic tangent profile, typical of free shear layers, has been shown to characterize canopy flows near the velocity inflection point at the top of the canopy (Raupach et al. 1996). Within experimental resolution, the inflection point coincides with the array edge, $y_{o} \approx 0$, except for the sparsest array $(\phi=0.02)$, for which $y_{o} \approx 2 d$ (see table 1). 
(a)

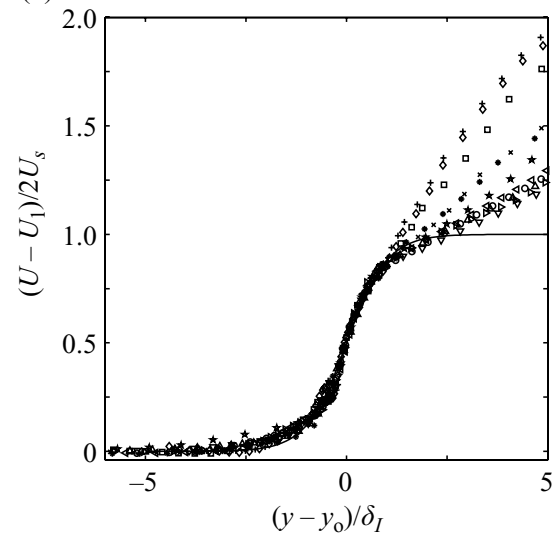

(b)

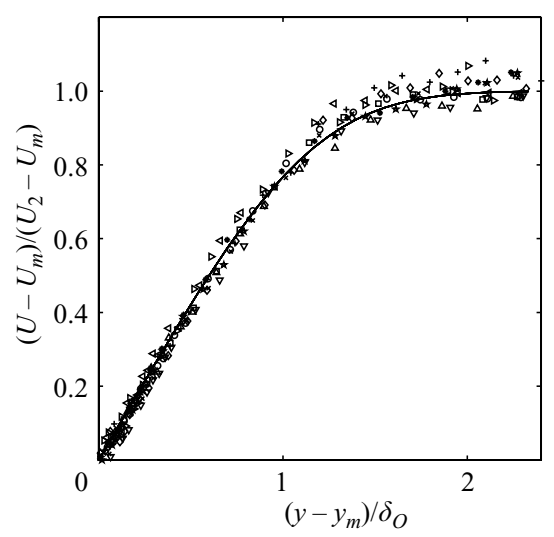

FIgURE 5. Two-layer scaling of the mean velocity. (a) Normalized spatially averaged innerlayer profiles for each experimental condition, with the hyperbolic tangent profile for reference (solid line). (b) Rescaled outer-layer profiles, with the Blasius boundary-layer solution for reference (solid line). The data (from table 1) are: $\square$, (I); $\diamond,(\mathrm{II}) ;+$, (III); $\times$, (IV); *, (V); $\star$, (VI); O, (VII); $\triangle$, (VIII); $\nabla,(\mathrm{IX}) ; \triangleleft,(\mathrm{X}) ; \triangleright,(\mathrm{XI})$.

In the outer layer, the velocity data are rescaled as $\left(U-U_{m}\right) /\left(U_{2}-U_{m}\right)$ and plotted against an outer-layer coordinate, $\left(y-y_{m}\right) / \delta_{O}$, where $y_{m}$, the effective boundary-layer origin, is the matching point between the inner region and the outer region and $U_{m}=U\left(y_{m}\right)$. This point is defined for each measured profile as the position at which the inner- and outer-layer slopes match. We determine $\delta_{O}$ for each velocity profile from the slope at the matching point,

$$
\delta_{O}=\frac{U_{2}-U_{m}}{\mathrm{~d} U /\left.\mathrm{d} y\right|_{y_{m}}}
$$

All parameters used in the scaling are labelled in figure $4(b)$.

Figure 5 shows the scaled inner- and outer-layer velocity profiles from all experimental cases. In the inner layer, the spatially averaged profiles collapse to an S-curve that nearly matches the hyperbolic tangent profile (5.3), which is shown as the solid line in figure $5(a)$. This profile, with its well-defined inflection point, is similar to the mixing-layer structure observed in canopy flows (Finnigan 2000; Ghisalberti \& Nepf 2002). However, unlike a classic shear layer, characterized by a single length scale, the full velocity profile is asymmetric. Thus, the inner-layer profiles follow the hypberbolic tangent in the inner layer (figure $5 a$ ), but diverge from the mixinglayer form as the outer region is approached, approximately $1.5 \delta_{I}$ outside the array edge. In the outer layer, the profiles collapse with the outer-layer scaling (figure $5 b$ ). Here, the profiles resemble a boundary-layer profile, and we have shown the Blasius boundary-layer solution (Schlichting 1979) for comparison (solid line). Note however, that the match to the Blasius profile does not imply that outer flow is identical to a developing laminar boundary layer, but simply that the mean velocity profile resembles a prototypical boundary-layer form. In summary, figure 5 clearly demonstrates that two independent length scales govern the fully developed shear layer.

In figure 6, the normalized Reynolds stress is plotted in inner- and outer-layer coordinates. Spatial fluctuations caused by cylinder wakes are apparent in the inner region and create some spread in the data (near $\eta_{I} \approx 0.5$ in figure $6 a$ ). Nonetheless, the inner layer collapses well and the Reynolds stress decays over a length of $\approx 2 \delta_{I}$. 
(a)

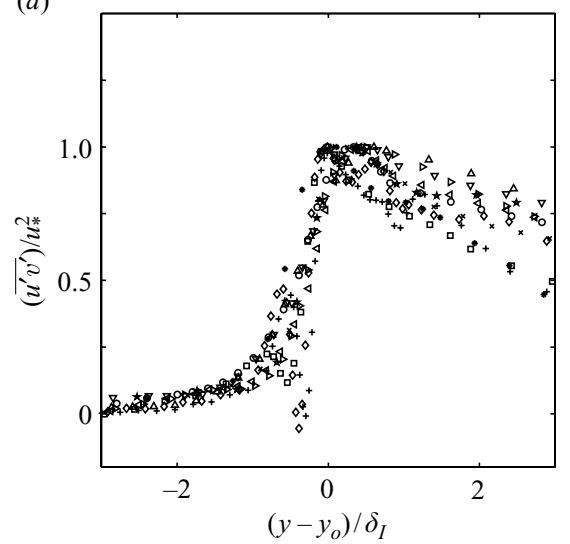

(b)

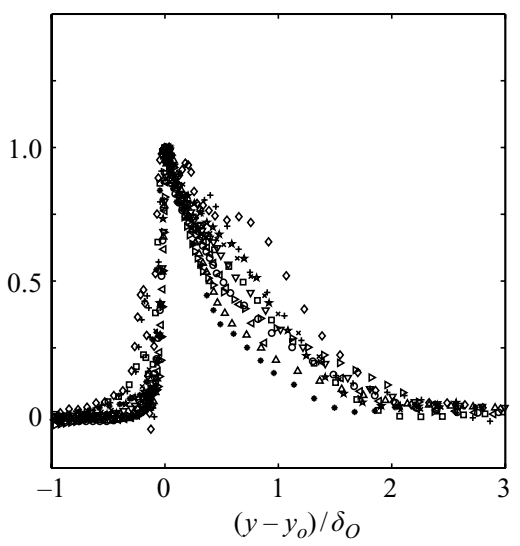

FiguRE 6. Two-layer scaling of the normalized Reynolds stress, $\left\langle\overline{u^{\prime} v^{\prime}}\right\rangle$. (a) Inner-layer scaling;

(b) outer-layer scaling. Symbols are as in figure 5.

$$
\begin{array}{lcc} 
& \delta_{O} & \left(C_{D} a\right)^{-1} \\
\delta_{I} & R^{2}=0.15 & R^{2}=0.87^{*} \\
h & R^{2}=0.76^{*} & -
\end{array}
$$

TABLE 2. Cross-correlations between relevant length scales $(n=11)$. Asterisks denote significance.

Reasonable collapse is seen in the outer region, and extension of $\left\langle\overline{u^{\prime} v^{\prime}}\right\rangle$ into the main channel scales with the outer-layer width, about $2 \delta_{O}$ for all cases, matching the extent of the velocity gradient (figure $5 b$ ).

Based on the scaling arguments described in $\S \S 3.3$ and 3.4, we expect the innerlayer width to be set by the length scale associated with array permeability, and the outer-layer width to be set by the water depth and bed friction coefficient. Those predictions were tested by linear regression across all experimental cases $(n=11)$ and the results are shown in table 2 . The length scales $\delta_{I}$ and $\delta_{O}$ are independent $\left(R^{2}=0.15, p=0.24\right)$, consistent with the two regions of collapse in figure 5. Moreover, each scaling prediction is confirmed. Specifically, $\delta_{I} \sim\left(C_{D} a\right)^{-1}\left(R^{2}=0.87, p=2 \times 10^{-5}\right)$, as predicted by (3.15), and $\delta_{O} \sim h / c_{f}\left(R^{2}=0.76, p=5 \times 10^{-4}\right)$, as predicted by (3.19). We have used a constant $c_{f}=0.005$ based on the vertical log-layer profiles measured in the open channel.

The inner-layer thickness is shown as a function of the array resistance length scale $\left(C_{D} a\right)^{-1}$ in figure 7. The regression line is shown, illustrating the $\delta_{I} \sim\left(C_{D} a\right)^{-1}$ scaling. However, we wish to call attention to the finite $y$-intercept, $\delta_{I}=0.92 \mathrm{~cm}$, or in terms of the cylinder diameter, $\delta_{I} \approx 1.5 d$. This intercept has physical significance, and represents the dense array limit discussed in $\S 3.3$, in which the roughness element diameter exceeds $\left(C_{D} a\right)^{-1}$ and thus sets the array penetration distance.

\section{Periodic fluctuations and instability}

The shear layer at the boundary of the cylinder array is dominated by coherent, nearly periodic fluctuations in the velocity and the free-surface elevation. 


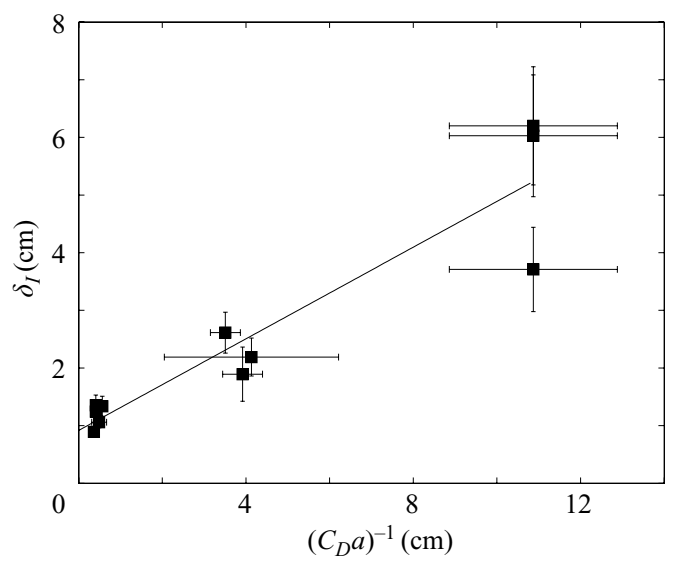

FIGURE 7. Inner-layer width, $\delta_{I}$ as a function of array resistance length scale, $\left(C_{D} a\right)^{-1}$. Solid line is the linear regression $\left(R^{2}=0.87, p=2 \times 10^{-5}\right)$.

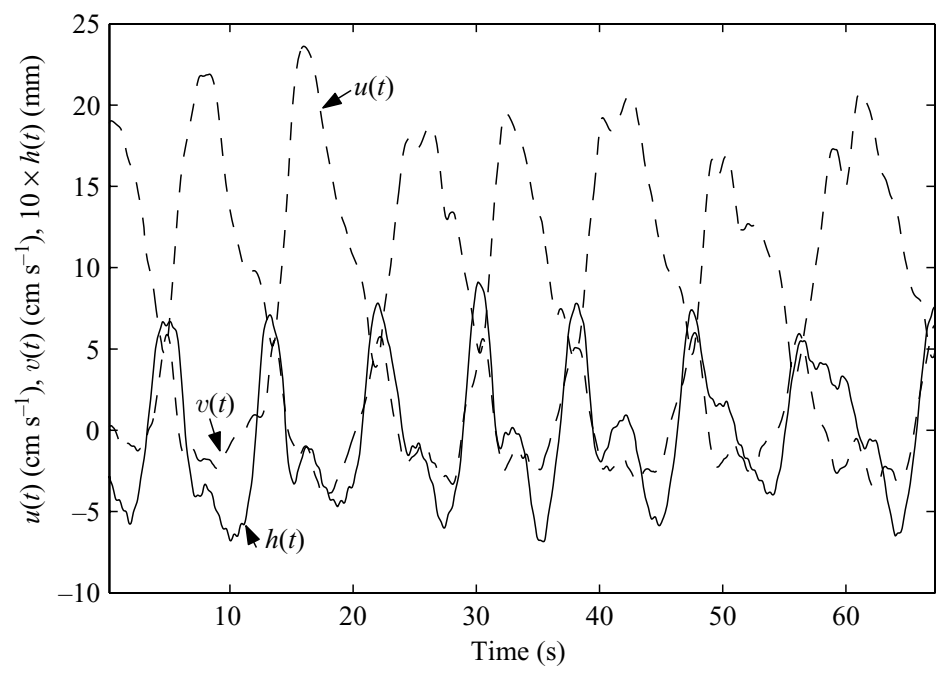

FIGURE 8. Temporal oscillations of the streamwise and transverse velocity and free-surface elevation for case X. $\phi=0.1, y=3 \mathrm{~cm}, x=6 \mathrm{~m}$.

Representative time series of the streamwise velocity, $u(t)$, the transverse velocity, $v(t)$, and the free-surface fluctuation, $h^{\prime}(t), 3 \mathrm{~cm}$ outside the interface are shown in figure 8 . The amplitude of the oscillations is significant relative to the mean, for example, the fluctuations in the streamwise velocity have root mean square intensity $u_{r m s} / U_{2} \approx 0.5$. The streamwise and transverse velocity fluctuations are anticorrelated, suggesting strong momentum transport events. Moreover, the free-surface displacement is in phase with the transverse velocity, demonstrating a pressure signature with the same period as the velocity fluctuations. The near periodicity and magnitude of the fluctuations demonstrate the presence of a single dominant frequency component, in contrast to the wide turbulence spectrum more common to open channel flows, and we can examine this component using spectral analysis.

Power spectral density plots of the transverse velocity fluctuations are shown for increasing position downstream of the splitter plate in figure 9. The frequency is 
(a)

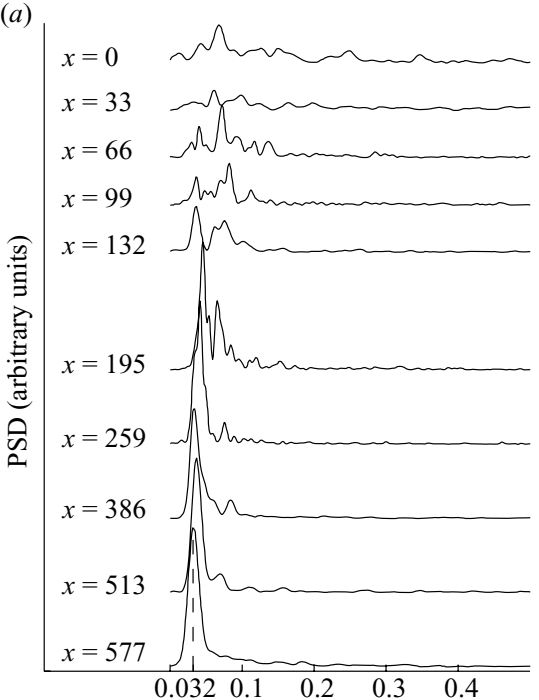

(c)

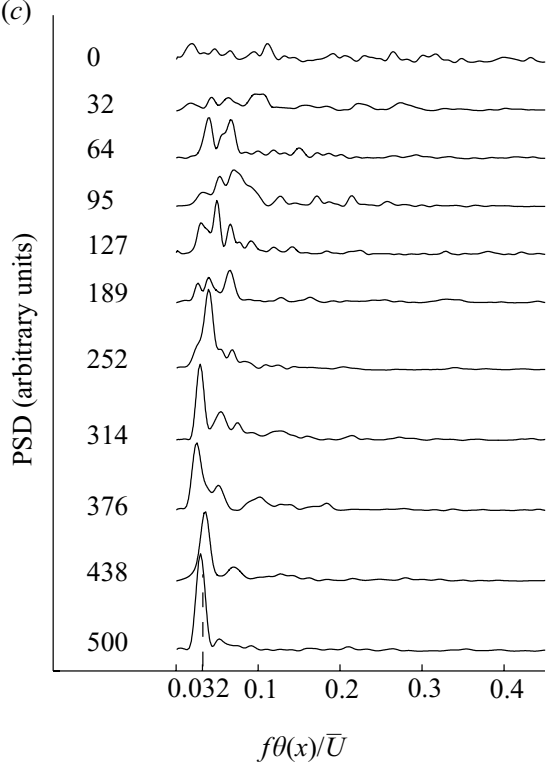

(b)

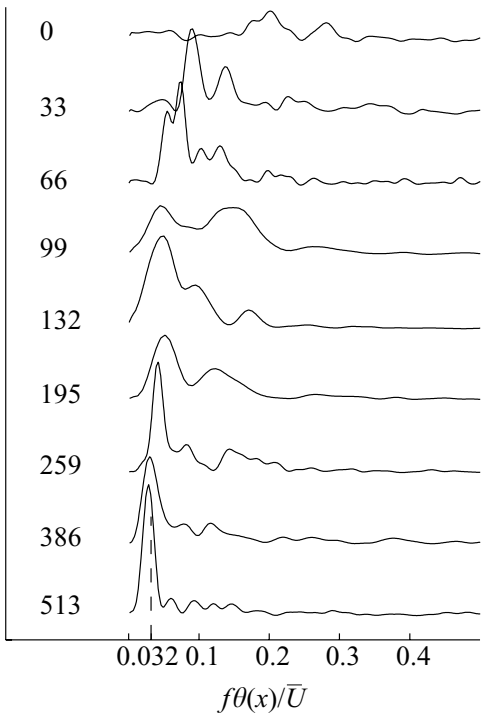

FIGURE 9. Development of the instability downstream of the splitter plate. The power spectral density for $v^{\prime}(t)$ is plotted for different $x$-positions (in $\mathrm{cm}$ ) for $(a)$ case $\mathrm{IV},(b)$ case $\mathrm{V}$ and (c) case VII. Abscissae for the PSD plots is the normalized frequency, $f \theta(x) / \bar{U}$. The natural frequency for a free shear layer, $f_{n} \theta / \bar{U}=0.032$ is shown by the dotted line. The magnitudes of the PSD curves are not shown, but each is scaled to be energy-preserving, i.e. $\int_{0}^{\infty} P_{v v} \mathrm{~d} f=\overline{v^{\prime 2}}$.

normalized by the local momentum thickness, $\theta(x)$, to form a dimensionless Strouhal number, $f \theta(x) / \bar{U}$. For each flow condition, a finite number of frequencies appear initially, typically with bimodal structure (see e.g. figure $9 b, x=33 \mathrm{~cm} ; 9 c, x=64 \mathrm{~cm}$ ), but sometimes exhibiting three or more modes (figure $9 b, x=66 \mathrm{~cm} ; 9 c, x=189 \mathrm{~cm}$ ). As the flow develops, energy is shifted to lower modes, consistent with the subharmonic instability commonly observed in free-shear-layer growth (Winant \& Browand 1974; Pierrehumbert \& Widnall 1982). Eventually an equilibrium is reached in which nearly 
all energy is contained in a single dominant frequency. This occurs by approximately the same longitudinal position $(x \approx 400)$ as the equilibrium of the mean velocity profiles (figure 3). The mean spectral peak for all flow conditions and array densities is $f_{d} \theta / \bar{U}=0.032 \pm 0.003$.

The coherent fluctuations are indicative of an instability of the Kelvin-Helmholtz type. In free shear layers possessing an inflection point, linear stability theory (Drazin \& Reid 1981) predicts the onset and growth of a shear instability which grows to a nonlinear state characterized by a row of coherent turbulent structures (see e.g. Brown \& Roshko 1974). The natural frequency of the passing structures is $f \theta / \bar{U}=0.032$ (Ho \& Huerre 1984), which precisely matches the dominant frequency for each case among the present experiments. This is particulary remarkable since the linear result is based on inviscid theory and a symmetric shear layer, as compared with our asymmetric partially obstructed shear layer. The key to the agreement is probably the equilibrium, and fixed $\theta_{e q}$, achieved in the partially obstructed layer, which allows the oscillation to lock into a fixed frequency. Given this lock-in, it is reasonable that the natural frequency of the shear flow is the most unstable linear mode.

An important distinction between the partially obstructed shear flow and a free shear layer is the downstream growth. A free shear layer grows continuously downstream, entraining irrotational fluid predominantly by vortex merging (Winant \& Browand 1974). However, in the partially obstructed shear layer, growth is inhibited by the resistance from the porous layer, leading to equilibrium. Equilibrium is also observed in submerged canopy flows, and vortices are observed at the linear frequency, $f \theta_{e q} / \bar{U}=0.032$ (Ghisalberti \& Nepf 2002). Shallow shear layers over a uniform rough bed can also experience arrested growth owing to bed friction. However, if the bed is uniform, there is no drag differential between the fast and slow streams to maintain the shear, and the shear layer is eventually erased and equilibrium is not attained (Uijttewaal \& Tukker 1998; Uijttewaal \& Booij 2000).

In our experiments, the fundamental frequency is set almost entirely by the outer-layer width associated with the main channel. To demonstrate, we rewrite the momentum thickness, $\theta$, as the sum of the inner-layer and outer-layer contributions,

$$
\theta=\theta_{I}+\theta_{O}=\int_{-\infty}^{y_{m}}\left[\frac{1}{4}-\left(\frac{U-\bar{U}}{\Delta U}\right)^{2}\right] \mathrm{d} y+\int_{y_{m}}^{\infty}\left[\frac{1}{4}-\left(\frac{U-\bar{U}}{\Delta U}\right)^{2}\right] \mathrm{d} y
$$

For all experimental cases, the ratio $\theta_{O} / \theta \geqslant 0.9$, which means that the frequency $f_{d}=0.032 \bar{U} / \theta$ is set primarily by the outer layer. This will be the case for most porous layers, provided that $\delta_{I} / \delta_{O} \ll 1$. Thus, although the instability originates at the inflection point within the inner layer, the inner-layer scale has little influence over the frequency once the instability is established. Similarly, Ghaddar et al. (1986a) studied oscillations near a groove-roughened wall that were initiated by a KelvinHelmholtz instability at the wall, but had a wavelength set by the boundary-layer mode for the channel, and Shvidchenko \& Pender (2001) observed regular vortices that scaled with the flow depth above permeable gravel beds. These channel-scale oscillations have been suggested as a mechanism for 'd-type' boundary-layer scaling near a rough wall, in which the effective roughness scales with the boundary-layer width rather than the roughness length (Djenidi, Anselmet \& Antonia 1994). 


\section{Coherent structure eduction}

Insight into the spatial structure of the instability was achieved by a vortex eduction technique based on conditional sampling of the LDV time series. For several of the experimental cases, a velocity profile sufficiently far downstream to be at equilibrium (typically $6 \mathrm{~m}$ ) was chosen for conditional sampling. Typically, vorticity or lateral velocity fluctuations are used as triggers for identifying coherent structures in shear flows (see, e.g. Bonnet et al. 1998). Here, we use zero crossings of the transverse velocity, $v(t)$. A vortex event was identified as lying between two successive zerocrossings of $v^{\prime}(t)$. In order to eliminate high-frequency fluctuations, $v(t)$ was first lowpass filtered below the dominant frequency, $f_{d}$. In the range $10<N<20$, structures were identified from the time series at each position. The conditionally averaged velocity at each position is obtained by an ensemble average over $N$ structures, following Hussain (1983):

$$
\langle\boldsymbol{u}\rangle_{c s}(\tilde{t})=\sum_{i=1}^{N} \boldsymbol{u}_{i}\left(t=\frac{T_{i}}{T_{d}} \tilde{t}+t_{z i}\right),
$$

where $\boldsymbol{u}_{i}(t)$ is the raw instantaneous LDA velocity vector between zero-crossings $i$ and $i+1, t_{z i}$ is the absolute time at which the $i$ th zero crossing occurs, $T_{i}$ is the period of the $i$ th structure, $T_{d}=1 / f_{d}$ is the mean period of all structures in the time series, and $\tilde{t}$ is the rescaled time, $\tilde{t} \in\left[0, T_{d}\right]$. That is, the structures are phase-aligned, stretched onto the $\left[0, T_{d}\right]$ grid, and then averaged. This removes small-scale fluctuations and phase jitter, but retains the large-scale structure. Note that although the filtered velocity is used to identify zero-crossings, the unfiltered velocity is used in the ensemble average.

For all cases, the $v(t)$ signal is in-phase to within $0.05 T_{d}$ across the entire shear layer, as determined from simultaneous two-point velocity measurements made using the LDV and an acoustic Doppler velocimeter. The negligible phase-lag of $v(t)$ allows the conditionally averaged velocity at each position to be aligned with the zero-crossing of the $v(t)$ signal, which yields the lateral structure. This is equivalent to

$$
\langle\boldsymbol{u}\rangle_{c s}(y, \tilde{t})=\sum_{i=1}^{N} \boldsymbol{u}_{i}\left(y, \frac{T_{i}}{T_{d}} \tilde{t}+t_{z i}+\phi(y)\right),
$$

where the phase is $\phi(y)=0$. Thus, $\langle u\rangle_{c s}(y, \tilde{t})$ and $\langle v\rangle_{c s}(y, \tilde{t})$ are the conditionally averaged velocity components over a single structure period. The time coordinate is then converted to the streamwise coordinate using the Taylor hypothesis. Zaman \& Hussain (1981) have noted that the Taylor hypothesis accurately reproduces the spatial dependence of velocity and vorticity when the structure passage speed is used for the time-space transformation everywhere across the shear layer. The structure passage speed, $U_{v}$, is measured from the two-point streamwise correlation of the surface displacement time series, which has the same period as the velocity fluctuations (figure 8). The streamwise coordinate in the moving frame of reference is then computed as, $x=\left(T_{d}-\widetilde{t}\right) U_{v}$.

\subsection{Conditionally averaged velocity field}

The conditionally averaged structure for a representative case (I) is shown in figure 10 . The structure is shown in the moving frame and the spatial coordinates are normalized by $\delta_{O}$. The structure is repeated to show more clearly the head of the trailing vortex and the tail of the preceding vortex. Figure 10(b) shows the sectional streamlines, which are tangent to the velocity vectors. A vortex centre $\left(x / \delta_{O} \approx 5\right)$ and two saddle points $(x \approx 0$ and $x \approx 10)$ are clearly visible, characteristic of the Kelvin cat's eye vortices 
(a)

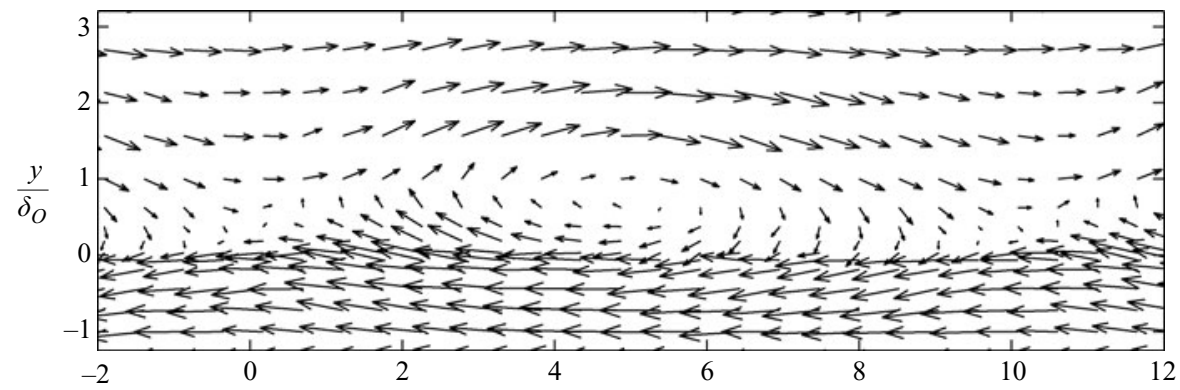

(b)

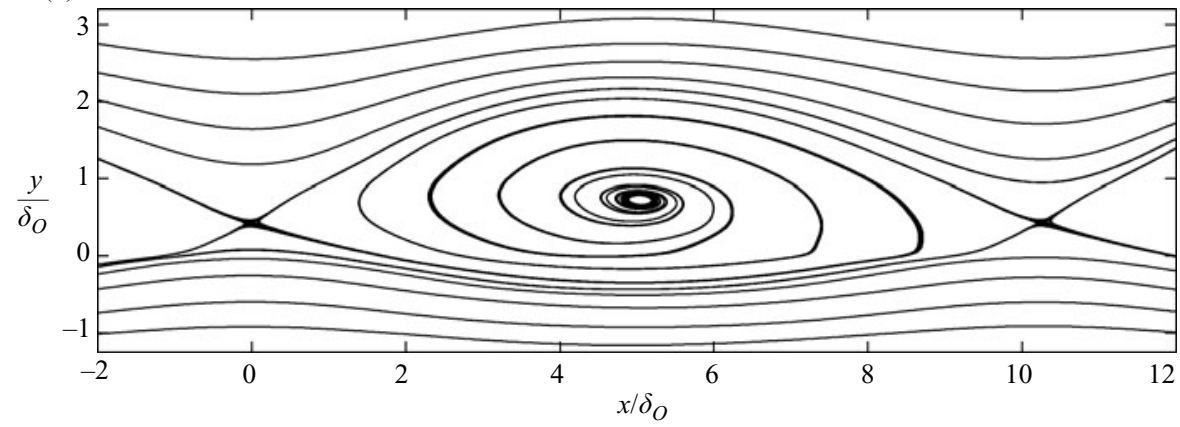

FIGURE 10. Structure of the conditionally averaged flow for case I, in a frame moving with the vortex. (a) Velocity vectors and $(b)$ sectional streamlines are shown for slightly more than one period of the vortex structure. Coordinates are normalized by $\delta_{O}$.

observed in free shear flows (Drazin \& Reid 1981). The primary vortex is defined as the region between the separatrices emanating from the saddle points (Perry \& Chong 1987). The vortex resides primarily in the open-flow region $(y>0)$ with a width of approximately $2 \delta_{O}$, and is elongated in the streamwise direction with a wavelength of $L_{v} \approx 10 \delta_{O}$. The length-to-width aspect ratio is 5 , slightly longer than vortices in free shear layers, which have aspect ratios of approximately 3 (based on the Stuart vortex model which fits experimental observations - see Browand \& Weidman 1976).

The topology around the vortex is an unstable focus, with trajectories spiralling outwards from the vortex centre. This pattern has been observed in other shallowwater vortices (Fu \& Rockwell 2005) and indicates the presence of a secondary circulation. To confirm this circulation, vortices were educed near the surface and near the bed (figure 11). Near the surface, the vortex rotation is outward spiralling, but near the bed the vortex spirals inward. This pattern indicates a three-dimensional circulation in which near-bed fluid is drawn toward the vortex centre and ejected at the surface. An upward axial flow would be required to conserve mass. This circulation is probably due to an imbalance between the radial pressure gradient and the tangential acceleration in the bottom boundary layer, resulting in a radial velocity component. We can estimate the contribution of the secondary stress as $\left\langle\left(\langle\bar{u}\rangle-\langle\bar{u}\rangle_{d}\right)\left(\langle\bar{v}\rangle-\langle\bar{v}\rangle_{d}\right)\right\rangle_{d}$ (see (3.8)). For this case, it is one order of magnitude smaller than the Reynolds stress across the region of shear. Thus, the secondary circulation appears to contribute little to the balance of angular momentum that governs the vortex dynamics. However, it may be significant for vertical transport of chemical species or biota in natural vegetated channels. 

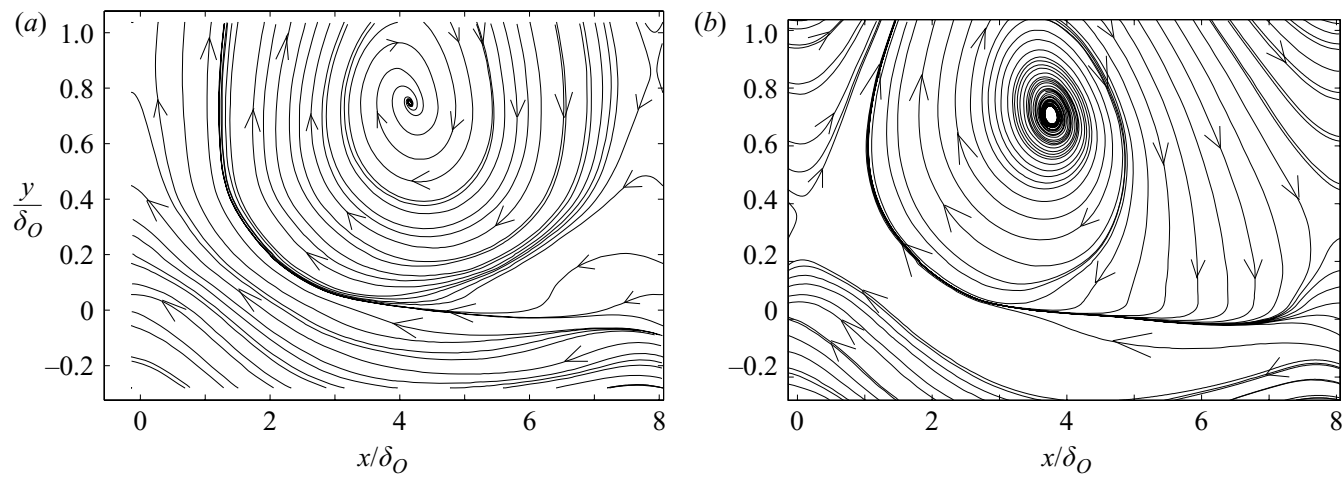

FIGURE 11. Sectional streamline plots in the horizontal plane at two depths (case $\mathrm{X}$ ): (a) $z=0.50 \mathrm{~cm} ;(b) z=4.0 \mathrm{~cm}$. In $(a)$, the circulation spirals toward the centre (stable focus); in $(b)$ it spirals away from the centre (unstable focus).
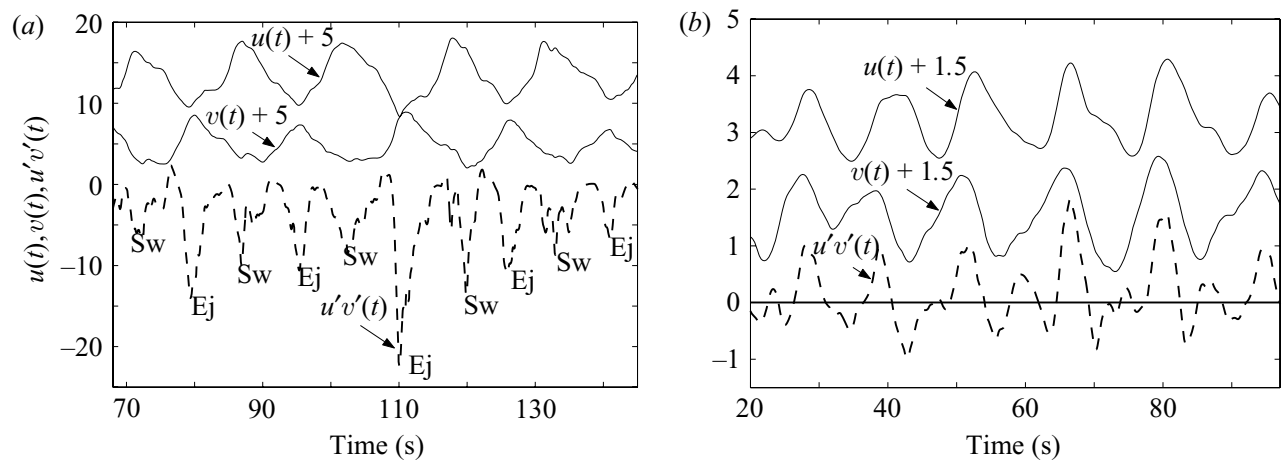

FiguRE 12. Time series of streamwise velocity, $u$, transverse velocity, $v$, (shifted for clarity) and instantaneous Reynolds stress, $u^{\prime} v^{\prime}$, at two locations: $(a)$ outside the interface, $y=3 \mathrm{~cm}$ and $(b)$ deep within the array, $y=-20 \mathrm{~cm}$. In $(a)$, sweeps $(\mathrm{Sw})$ and ejections $(\mathrm{Ej})$ are labelled.

\subsection{Conditionally averaged Reynolds stress}

The vortices create strong velocity fluctuations, both within and away from the central core. Here we examine the effect of these fluctuations on lateral momentum flux. Outside the central core, the streamlines undulate, but are not rotational (figure 10), indicating a wavelike oscillatory flow both within the array and in the open channel outside the shear layer. Figure 12 compares velocity time series at $y=3 \mathrm{~cm}$, within the vortex core (figure 12a) and at $y=-20 \mathrm{~cm}$, outside the vortex core but deep within the array (figure 12b). At both locations the regular velocity fluctuations, $u^{\prime}$ and $v^{\prime}$, correspond to the dominant frequency, $f_{n}$. Within the vortex (figure 12a), there are strong velocity correlations, $u^{\prime} v^{\prime}$, corresponding to momentum inflows, termed sweeps $\left(u^{\prime}>0, v^{\prime}<0\right)$, and momentum outflows, termed ejections $\left(u^{\prime}<0, v^{\prime}>0\right)$. These events, which transport momentum across the interface, contribute to the large time-averaged Reynolds stress, $\overline{u^{\prime} v^{\prime}}$, observed outside the array (see figure $3 b$ ). In contrast, outside the vortex core and deep within the array (figure $12 b$ ) the regular oscillations in $u^{\prime}$ and $v^{\prime}$, are approximately out of phase, so that the positive and negative momentum fluxes cancel, resulting in a very small net Reynolds stress, $\overline{u^{\prime} v^{\prime}} \approx 0$. The vortices, 

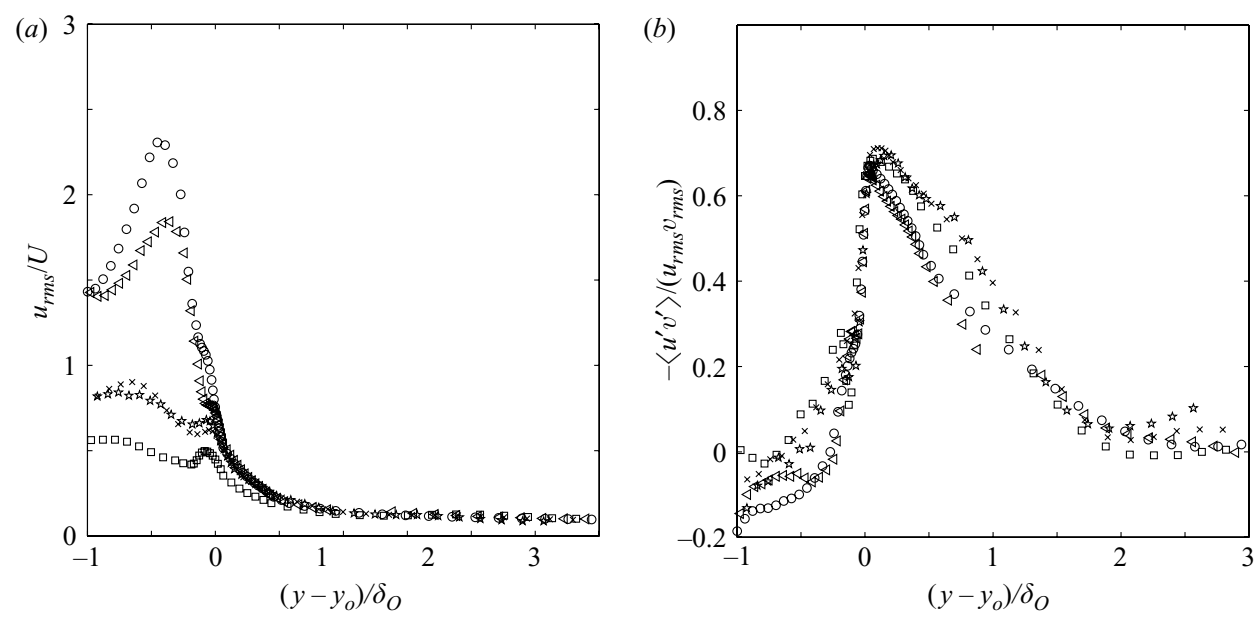

FIGURE 13. (a) Strength of the velocity fluctuations relative to the local flow, $u_{r m s} / U$ and $(b)$ Reynolds stress correlation coefficient, $-\left\langle\overline{u^{\prime} v^{\prime}}\right\rangle / u_{r m s} v_{r m s}$ for a range of cylinder density: case I, $\phi=0.02(\square)$; cases IV and VI, $\phi=0.045(\times, \star)$; cases VII and X, $\phi=0.1(\bigcirc, \triangleleft)$.

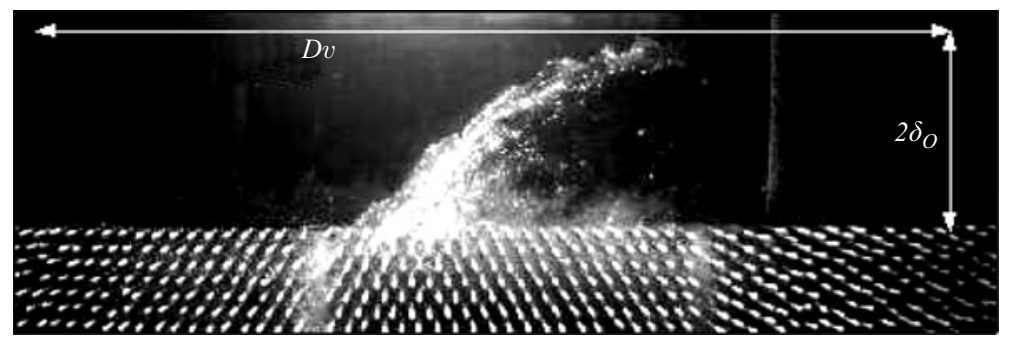

FIGURE 14. Visualization of an ejection event (mean flow is left to right).

induce this far-field wave response through the passage of alternating low-pressure cores and high-pressure regions (see, e.g. figure 8). The pressure field sets up a passive wave response within the array, but does not create substantial momentum flux. The wave response is also present in the velocity time series in the channel outside the region of shear $\left(y \gtrsim 2 \delta_{o}\right)$. We do not interpret these coherent motions as turbulence, but rather as coherent vortices coupled with a wave response.

Within the array, the velocity fluctuations are large compared with the mean velocity (figure $13 a$ ), which shows $u_{r m s} / U$ for different cylinder fractions. For the most dense array $(\phi=0.1), u_{r m s} / U$ is well above unity for $y<0$. This suggests that the fluctuations induced by the passing vortices are sufficiently strong to reverse the flow; this was confirmed by flow visualization. However, the correlation coefficient, $\overline{u^{\prime} v^{\prime}} / u_{r m s} v_{r m s}$, which measures the momentum transport efficiency of the fluctuations, is very small within the array, consistent with the wave response (figure 13b). Outside the array interface, where the vortex resides, the correlation is maximized, indicative of the strong momentum transport by the vortices.

To illustrate the momentum transport induced by the vortices, an ejection event, visualized by $10 \mu \mathrm{m}$ reflective particles, is shown in figure 14 . The image illustrates that the vortex motions are responsible for significant fluxes, and can transport mass and momentum over length scales of approximately $2 \delta_{O}$. 


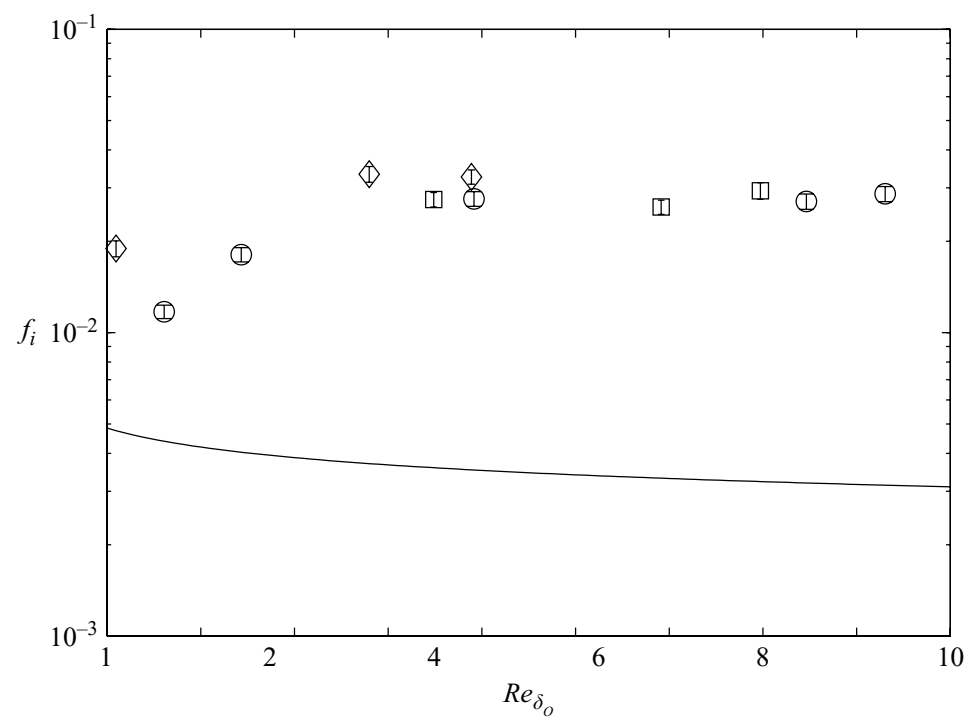

FIGURE 15. Friction coefficient at the array interface, $f_{i}=u_{*}^{2} /\left(\Delta U^{2} / 2\right) v$ s. the channel Reynolds number, $\operatorname{Re}_{\delta_{O}}=2 \delta_{O} \Delta U / v$ for all experimental profiles: $\phi=0.02(\square), \phi=0.045(\diamond)$, and $\phi=0.1$ (०). Solid line is the friction coefficient, $c_{f}=u_{*}^{2} /\left(U_{o}^{2} / 2\right)$ for a turbulent solid wall (Pope 2000, p. 278).

The interfacial friction coefficient, $f_{i}=u_{*}^{2} /\left(\Delta U^{2} / 2\right)$, is a measure of the momentum exchange across the interface. From figure 15 , the friction coefficient values observed in these experimental cases are nearly an order of magnitude larger than the values observed near a solid wall (Pope 2000, p. 278). The enhanced momentum exchange at the porous wall is attributed to the coherent structures, which are absent at a solid wall. Mass transport across the interface will be similarly enhanced.

The conditionally averaged Reynolds stress, $-\left\langle u^{\prime} v^{\prime}\right\rangle_{c s}(x, y)$ (calculated using (7.1)), gives an insight into the spatial structure of the momentum transport. Figure 16 shows $-\left\langle u^{\prime} v^{\prime}\right\rangle_{c s}$, normalized with $u_{*}^{2}$, for each cylinder density. In each case, the maxima of $-\left\langle u^{\prime} v^{\prime}\right\rangle_{c s}$ are located in regions of strong crossflow, the outflow region $\left(1<x / \delta_{O}<4\right)$ and the inflow region $\left(6<x / \delta_{O}<9\right)$, just outside the array interface. These regions correspond to the ejections and sweeps, respectively, and are responsible for the instantaneous momentum fluxes observed in figure 12(a). The ejections account for greater net momentum transport (higher $u^{\prime} v^{\prime}$ maximum) than sweeps. The sweeps and ejections are confined to a region approximately between the array interface and about $y=2 \delta_{O}$, corresponding to the width of the central vortex (figure 10). In addition, the net Reynolds stress is appreciable only within this same region (see adjacent plots of $\left\langle\overline{u^{\prime} v^{\prime}}\right\rangle$ ). Outside the central vortex, both within the array and in the free stream, $-\left\langle u^{\prime} v^{\prime}\right\rangle_{c s}$ has a banded appearance, consisting of alternating positive and negative Reynolds stress. These alternating bands correspond to the far-field wave response, and the positive and negative fluxes cancel when averaged over the cycle. It can be verified from the adjacent plots that $\left\langle\overline{u^{\prime} v^{\prime}}\right\rangle \approx 0$ in these regions. Overall, the structure of the Reynolds stress is nearly identical across array density, $\phi$, consistent with the idea that the vortex characteristics, and momentum transport, are established by the outer layer. 

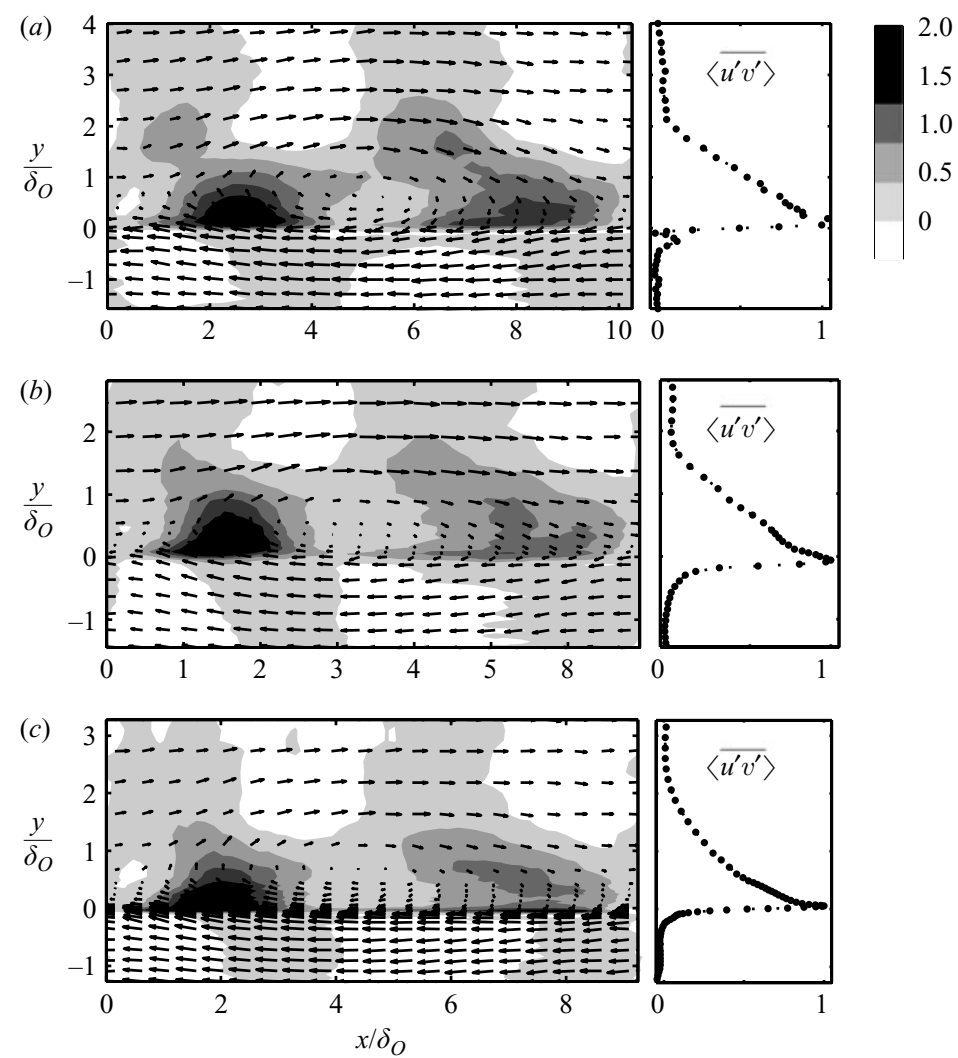

FIGURE 16. Contours of the conditionally averaged Reynolds stress, $-\left\langle u^{\prime} v^{\prime}\right\rangle_{c s} / u_{*}^{2}$, with velocity vectors over one cycle for each cylinder density: $(a)$ case I, $\phi=0.02,(b)$ case IV, $\phi=0.045$, and (c) case VII, $\phi=0.1$. Areas of white represent $-\left\langle u^{\prime} v^{\prime}\right\rangle_{c s}<0$. Corresponding mean Reynolds stress, $-\left\langle\overline{u^{\prime} v^{\prime}}\right\rangle / u_{*}^{2}$, is shown adjacent. The grey scale is the same for all plots.

\subsection{Conditionally averaged vorticity}

Figure $17(c)$ shows the vorticity distribution of the conditionally averaged vortex,

$$
\omega=\frac{\partial\langle v\rangle_{c s}}{\partial x}-\frac{\partial\langle u\rangle_{c s}}{\partial y},
$$

which has been normalized by the scale for interfacial shear, $U_{s} / \delta_{I}$. This particular normalization was chosen because the interface is the region of maximum shear and the dominant source of vorticity. There is a thin line of high-vorticity fluid near the interface, $y=0$, at the vortex front between $x / \delta_{O} \approx 7$ and $x / \delta_{O} \approx 11$. This coincides with the region of maximum shear and occurs in the region dominated by sweeps from the outer region. A second region of high-vorticity fluid takes the form of a tongue between $x / \delta_{O} \approx 1$ and $x / \delta_{O} \approx 6$. This tongue appears to be ejected from the array interface into the outer layer. A second tongue of nearly irrotational fluid, from $x / \delta_{O} \approx 4$ and $x / \delta_{O} \approx 9$, is sandwiched between the two high-vorticity regions. The alternating high- and low-vorticity regions can be explained by the inherent asymmetry of the partially obstructed shear layer. The low-vorticity fluid has its origin in the free stream where the mean shear $(\partial U / \partial y)$ is weak, whereas the high-vorticity fluid originates in the high-shear interfacial region. This shear asymmetry is not present, for example, in free shear layers for which the velocity profile is antisymmetric 

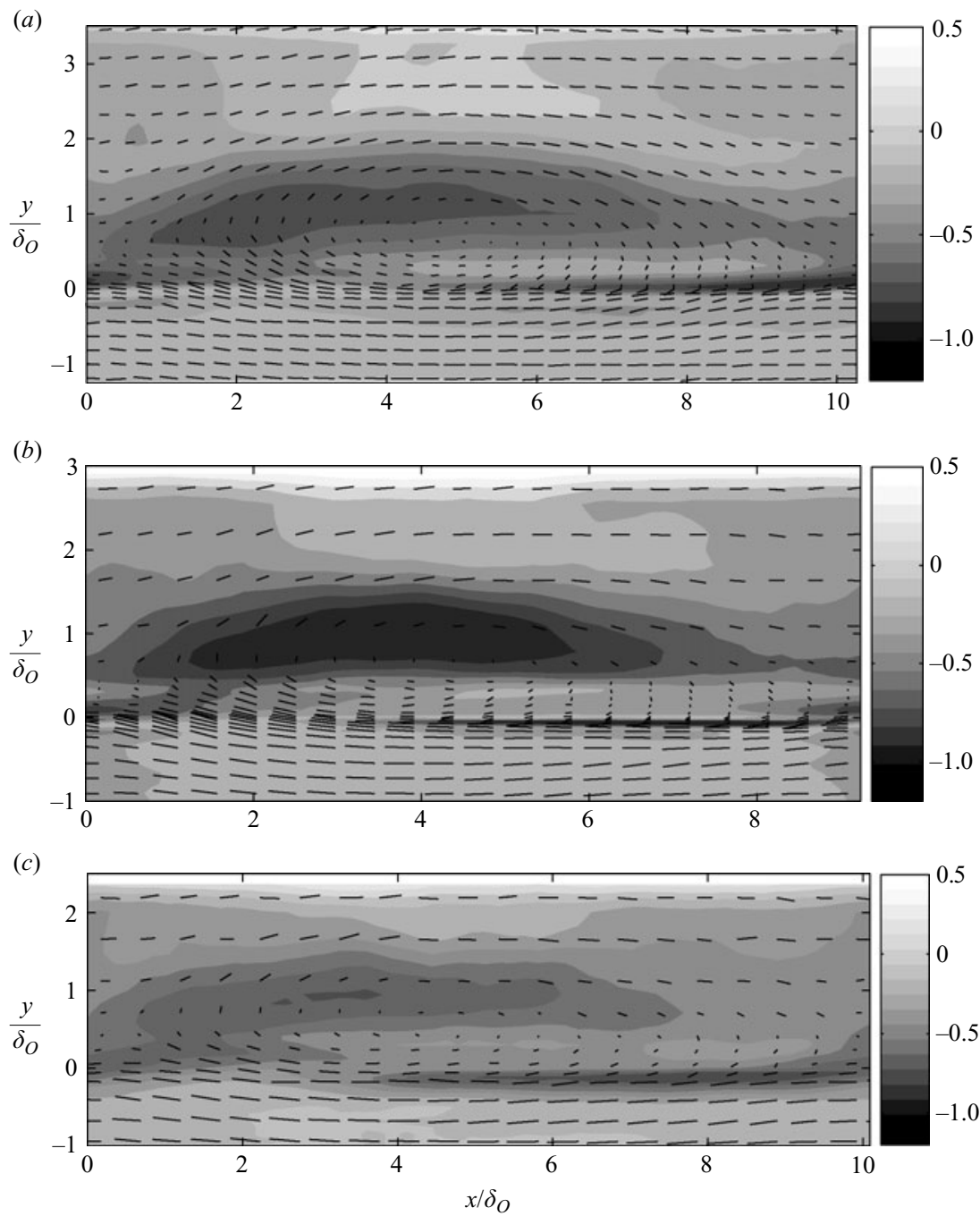

FIGURE 17. Vorticity contours of the conditionally averaged vortex for $(a)$ cases I, (b) VII, and (c) V. Velocity vectors are shown for reference.

with only one length scale, and the vorticity is approximately symmetric about the centreline.

7.4. Conditionally averaged kinetic energy production and dissipation

The kinetic energy production associated with the coherent structures is given by

$$
\langle P\rangle_{c s}=-\left\langle u^{\prime} v^{\prime}\right\rangle_{c s} \frac{\partial\langle u\rangle_{c s}}{\partial y} .
$$

The spatial distribution of $\langle P\rangle_{c s}$ for case I is shown in figure 18. Also shown for reference is the wavelength-average (or time-averaged) production, $\bar{P}$. Nearly all production occurs near the inflection point at the interface, where both the shear and Reynolds stress are large. The production maximum lies in the sweep region along a very thin line between $x / \delta_{O} \approx 4$ and $x / \delta_{O} \approx 6$ and corresponds to the sharp peak in $\bar{P}$ at the interface. The production is less pronounced in the ejection region, where the 

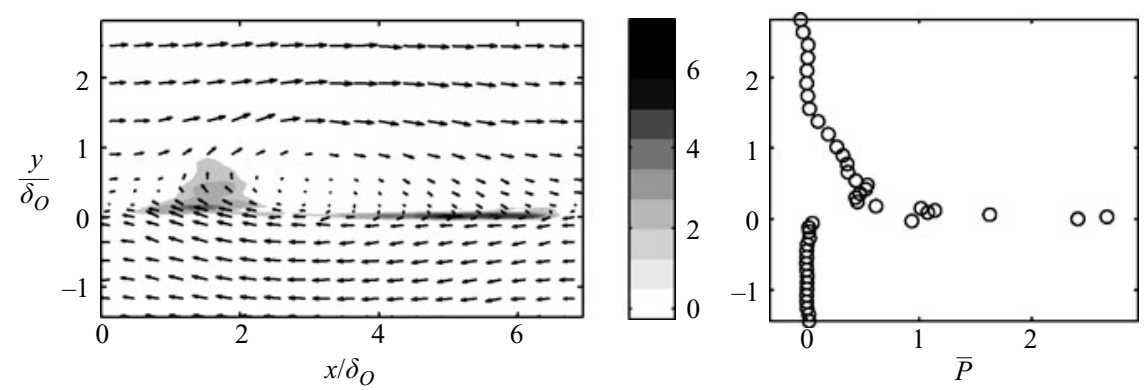

FIGURE 18. Contours of the coherent structure kinetic energy production, $\langle P\rangle_{c s}$ normalized by $u_{*}^{3} / \delta_{I}$, for case III. Also shown is the time-averaged production, $\bar{P}$.
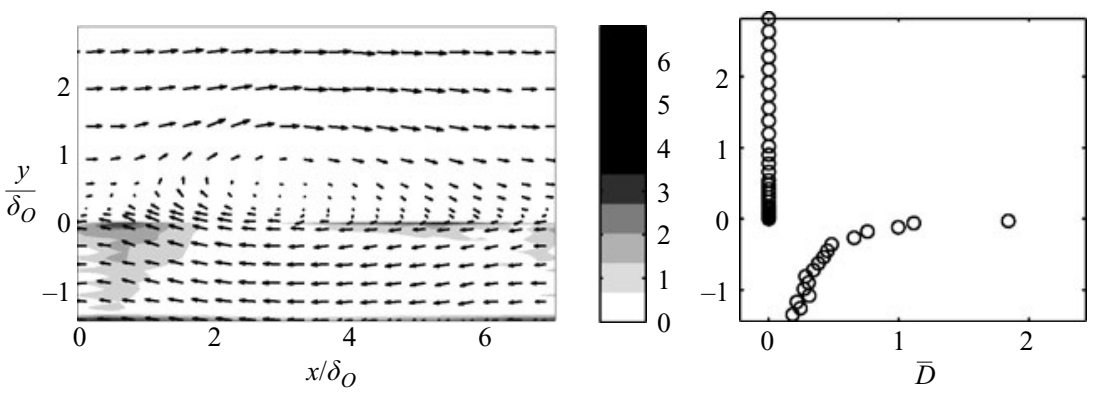

FIGURE 19. Contours of the dissipation of coherent structure kinetic energy by array drag, $\langle D\rangle_{c s}$ normalized by $u_{*}^{3} / \delta_{I}$, for case III. Also shown is the time-averaged dissipation, $\bar{D}$. Note that the discontinuity in $\bar{D}$ is due to the drag discontinuity across the array interface.

Reynolds stress is maximum but the velocity gradient is smaller. This indicates that sweeps play the dominant role in maintaining the coherent structures, by carrying high-momentum fluid toward the interface and creating very high shear, $\partial U / \partial y$.

The dissipation of kinetic energy by the cylinder array is given by

$$
\langle D\rangle_{c s}=\frac{1}{2} C_{D} a\left(\langle u\rangle_{c s}^{2}+\langle v\rangle_{c s}^{2}\right)^{3 / 2}
$$

where $\langle u\rangle_{c s}$ and $\langle v\rangle_{c s}$ are the conditionally averaged velocities in the laboratory frame of reference. The spatial distribution of $\langle D\rangle_{c s}$ and the lateral dependence of the timeaveraged value, $\bar{D}$, are shown in figure 19 . The greatest dissipation is concentrated near the interface, where the in-array velocities are highest. Both the spatial pattern and the magnitude of the dissipation is comparable with the production (figure 18), and the time-averaged values are approximately equal near the interface, $\bar{P} \approx 2.5$ and $\bar{D} \approx 2$. This suggests the kinetic energy production at the interface is approximately balanced by the dissipation due to array drag. This balance is a key to the vortex equilibrium observed in experiments, suggesting that the vortex growth is quenched when production balances dissipation. However, another important term in the total energy budget is the pressure-strain term, $-\partial_{y}\left[\langle v\rangle_{c s}\langle p\rangle_{c s}\right]$, the work done by the vortex pressure field in driving lateral flow through the obstructed array. Although this term is difficult to measure, and not shown here, it is an energy sink and can be expected to contribute, along with the drag dissipation, to balancing the energy production. The importance of this term near a permeable wall is demonstrated in the DNS results of Breugem et al. (2006). 


\section{Discussion}

Taken together, the structure of the Reynolds stress, vorticity and energy production give an insight into the cycle that produces and maintains the coherent structures. The high-vorticity fluid along the interface is created by sweeps that transport highmomentum channel fluid toward the array, enhancing the velocity gradient (figure 17). The resulting peak in Reynolds stress and velocity gradient leads to very high local energy production which feeds the shear instability and maintains the vortices. The ejections then carry the high-vorticity fluid from near the interface back into the main channel, leading to substantial momentum transport. However, it is the sweeps that appear to be the key to the vortex production, since they create the high shear at the interface that leads to high energy production (figure 18).

It is natural to focus on the sweeps and their enhancement of interfacial shear, because this process reinforces the velocity inflection point, which leads to the shear instability. Moreover, while the instability originates in the inner layer, the characteristics of the vortices - their dimensions and the structure of the sweeps scale with the outer layer $O\left(\delta_{O}\right)$. In the cases we studied, the mean array drag, $C_{D} a$, changed by an order of magnitude, but the vortex characteristics in the outer region were nearly identical. Nonetheless, the role of the array is essential, and has two direct effects: (i) the drag discontinuity at the interface creates large-scale shear which leads to instability and coherent vortices and (ii) the array resistance reinforces the interfacial shear by limiting vortex penetration, and causing high-momentum fluid to build up at the interface. Moreover, drag acts on the portion of the vortex that lies within the array, leading to dissipation of kinetic energy (see figure 19) (and also of vorticity), and limiting the vortex penetration to the inner-layer width, $\delta_{I}$. However, the total drag acting on the portion of the vortex within the array is proportional to $C_{D} a \delta_{I}$, which was approximately constant across all cases (see $\$ 5.2$ ). It appears that the flow adjusts until the vortex reaches its maximum penetration (determined by the array resistance, $C_{D} a$ ), after which, further growth is quenched by drag (see figure 3). A balance is thus maintained between the kinetic energy production, driven by the outer-layer sweeps, and the dissipation by the array drag, which occurs in the inner layer.

As discussed previously, the contribution of three-dimensional effects to the dynamics of the instability appears to be small, as the secondary circulation contributes little to the overall stress balance. Moreover, the energetic cycle hypothesized to maintain the vortices is essentially two-dimensional. Although three-dimensional turbulence and secondary circulations may modify the details of the coherent structures, they do not appear to be intrinsic to the instability. This is further supported by two-dimensional LES simulations of partially vegetated channels which successfully captured vortex oscillations (Nadaoka \& Yagi 1998; Xiaohui \& Li 2002) similar to those we observed. Moreover, these authors used a simple quadratic drag model for the vegetation layer, suggesting that the details of the porous layer, such as cylinder arrangement, are relatively unimportant beyond setting the bulk drag. These results are consistent with the energetic cycle we have proposed, which requires only a penetrable roughness layer in order to create velocity inflection at the interface and promote ejections. They are also consistent with the scaling of the observed vortex frequency and size with the outer layer, which suggests array details are relatively unimportant. A linear stability analysis in the next section will further support our conclusion that the instability is both two-dimensional and sensitive only to the bulk resistance of the porous layer. 


\section{Linear stability analysis}

The experimental results demonstrate that the drag differential between the array and the channel leads to shear instability. However, the presence of drag can also have a stabilizing effect, as observed in shallow shear layers with strong bed friction $(\mathrm{Chu}$, Wu \& Khayat 1991; Uijttewaal \& Booij 2000). To assess these competing influences, a linear stability analysis was carried out for the growth of perturbations to the mean velocity profile in the presence of drag. The mean velocity is approximated by a hyperbolic tangent profile with the inflection point at the interface,

$$
U(y)=U_{o}(1+R \tanh (y / b)),
$$

where $U_{o}$ is the centreline velocity, $b$ is the shear-layer width, and $R$ is the velocity difference between the streams far outside the shear layer,

$$
R=\frac{U_{2}-U_{1}}{U_{2}+U_{1}} .
$$

This antisymmetric profile is a good approximation for the sheared region near the inflection point at the onset of instability, when the perturbation amplitude is small. Further downstream, the disturbance grows in a nonlinear way and reaches the finiteamplitude equilibrium observed in experiments, but this nonlinear regime is beyond the scope of the present theory.

We assume that the drag is quadratic in velocity and consists of a uniform bed friction to which array drag is added on one side of the channel as in (3.8c). The drag differential between the porous layer and the main channel, can be parameterized by the non-dimensional number

$$
\gamma=\frac{C_{D} a}{C_{D} a+2 c_{f} / h} .
$$

The mean resistance, composed of both array and bed drag, is measured by the nondimensional parameter

$$
S=\left(C_{D} a / 2+c_{f} / h\right) b,
$$

termed the stability number in the shallow-flow literature (see, e.g. Chu et al. 1991; Socolofsky \& Jirka 2004).

It is reasonable to assume that outside the region of shear, the mean pressure gradient is in balance with the local drag, which implies

$$
\frac{1}{2} \rho\left(C_{D} a+c_{f} / h\right) U_{1}^{2}=\frac{1}{2} \rho\left(c_{f} / h\right) U_{2}^{2},
$$

from which it can be shown that $\gamma$ and $R$ are related by

$$
R=\frac{1}{\gamma}\left(1-\sqrt{1-\gamma^{2}}\right) .
$$

Thus, for sparse arrays for which $C_{D} a \rightarrow 0$, the shear vanishes and $R \rightarrow 0$. However, as the array resistance increases, the velocity difference across the shear layer also increases. This shear enhancement can be expected to favour instability. On the other hand, an increase in total drag, through increasing $S$, will tend to damp instability. Our objective is to determine the stability characteristics for different array densities by varying the drag differential, $\gamma$, and the overall friction, $S$.

We begin with the equations of motion for a two-dimensional shallow flow and assume that the Reynolds number based on the momentum thickness is large, $R e_{\theta} \gg$ 1 , such that the flow is assumed inviscid beyond the effect of drag. Following classic 
theory (see e.g. Drazin \& Reid 1981), a two-dimensional disturbance is introduced to the mean velocity and pressure distributions,

$$
u=U(y)+u^{\prime}(x, y, t), \quad v=v^{\prime}(x, y, t), \quad p=P(y)+p^{\prime}(x, y, t) .
$$

and written in normal mode form,

$$
u^{\prime}=\phi_{u}(y) \exp (\mathrm{i}(k x-\omega t)), v^{\prime}=\phi_{v}(y) \exp (\mathrm{i}(k x-\omega t)), p^{\prime}=\phi_{p}(y) \exp (\mathrm{i}(k x-\omega t)) .
$$

The substitution of the disturbance into the shallow-water momentum equation (3.7) yields a modified Rayleigh equation (MRE) (Chu et al. 1991; Chen \& Jirka 1997; Socolofsky \& Jirka 2004),

$$
(c-U) \phi_{y y}+\left(U_{y y}+k^{2}(U-c)\right) \phi=\frac{S H(y) U}{\mathrm{i} k}\left(\phi_{y y}+\frac{U_{y} \phi_{y}}{U}-\frac{k^{2} \phi}{2}\right),
$$

where the eigenfunction, $\phi \equiv \phi_{v}$, satisfies the boundary conditions,

$$
\phi \rightarrow 0 \text { as } y \rightarrow \pm \infty,
$$

$c=\omega / k$ is the wave speed, and $H(y)$ is a Heaviside step function, given by

$$
H= \begin{cases}1+\gamma, & y<0 \\ 1, & y=0 \\ 1-\gamma, & y>0\end{cases}
$$

The friction term, multiplied by the factor $S H(y)$, tends to damp the instability.

Equations (9.9)-(9.11) form an eigenvalue problem yielding a dispersion relationship, $F(\omega, k, S, \gamma)=0$. Since we are interested in the downstream disturbance growth, often termed the spatial instability problem, the eigenvalue problem is solved for the complex wavenumber, $k=k_{r}+i k_{i}$; a disturbance of frequency $\omega$ is amplified if $k_{i}<0$. The eigenvalue problem was solved using a pseudospectral collocation method with Chebyshev polynomials. Calculations were made using the program suite SWESC-M (Shallow Water Eigenvalue Stability Calculator - Matlab) developed by S. A. Socolofsky. Details are given in Socolofsky \& Jirka (2004).

As a check of the numerical method, we first present results for uniform bottom friction, $(\gamma=0)$ (figure 20), but for which a velocity difference $(R=1)$ is imposed, as in Chu et al. (1991). The frictionless, $S=0$, case is identical to the results of Monkewitz \& Huerre (1982) for a free shear layer. The most unstable frequency occurs at $f \theta / \bar{U} \approx 0.032$. As the drag is increased, by increasing $S$, the growth rate decreases uniformly across the frequency spectrum. When $S \approx 0.25$, all modes becomes stabilized $\left(k_{i} \approx 0\right)$. The frequency of the most unstable mode changes very little even as all modes are progressively damped. These results match those of Chu et al. (1991) for a shallow shear layer with uniform friction.

Calculations were made with discontinuous drag to assess the competing tendencies of increasing drag to damp shear instability and increasing drag differential to promote it through enhanced shear. Figure 21 shows the neutral stability curve, i.e. the critical friction number, $S_{c}$, at which the growth rate of the most unstable mode is reduced to zero, as a function of the drag differential, $\gamma$. When the drag differential, $\gamma$, is small, the critical value, $S_{c}$, is small and increasing the drag only tends to damp the instability. However, as $\gamma \rightarrow 1, S_{c} \rightarrow \infty$; thus, increasing drag leads to instability by increasing the drag differential which enhances the shear. In this limit, the stabilizing effect of friction is overcome by the drag differential. Note that the $\gamma=1$ case is 


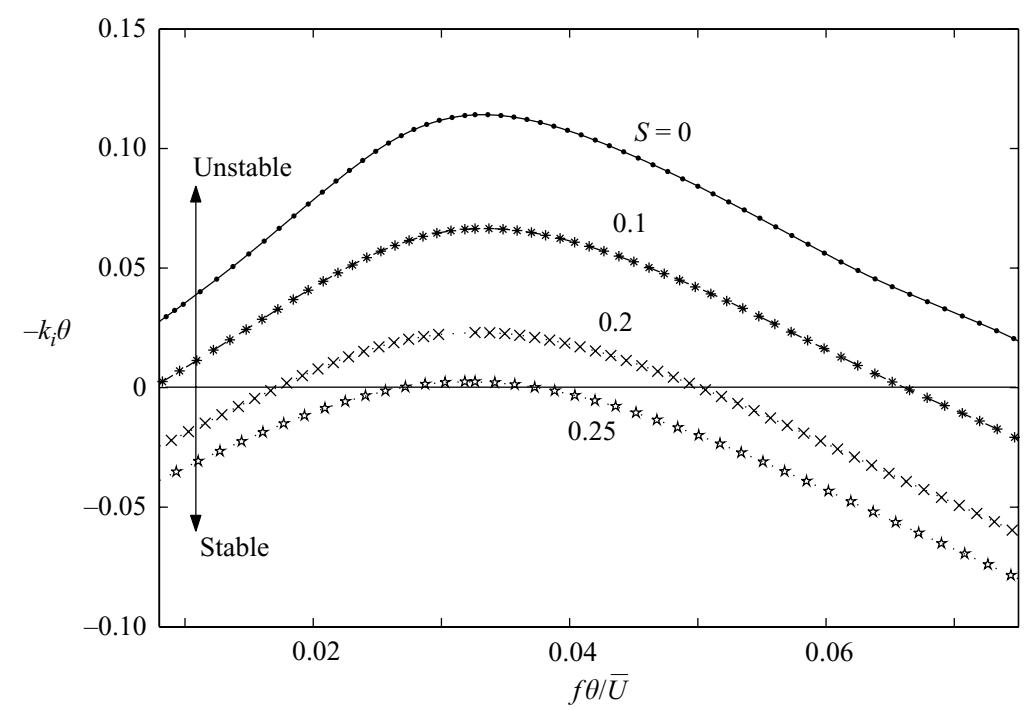

FIGURE 20. Spatial growth rate for uniform drag $(\gamma=0)$ and various values of the friction number.

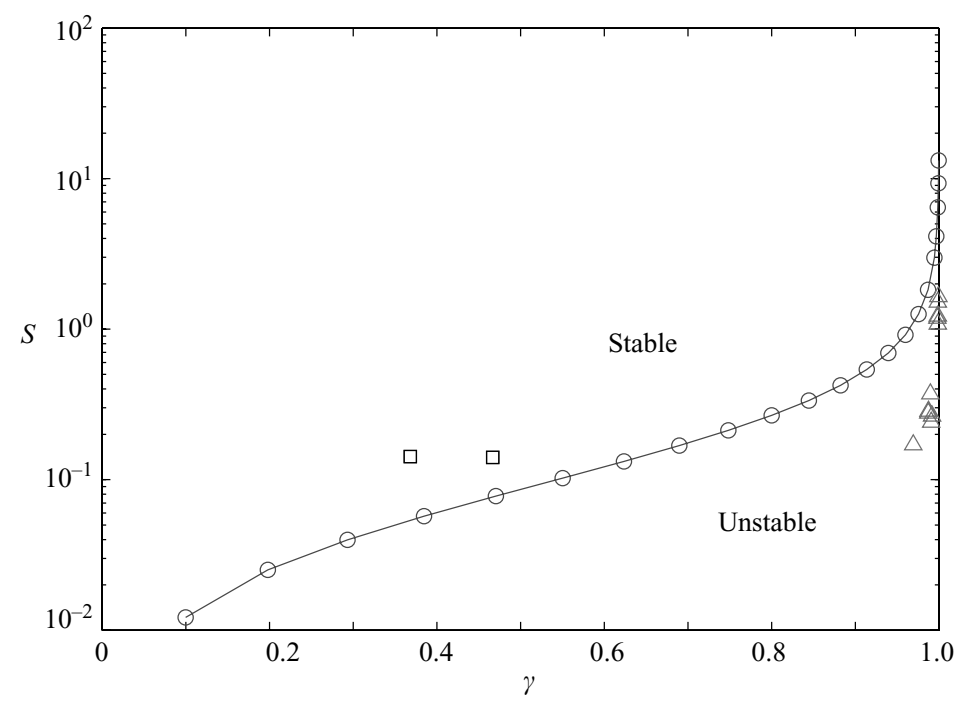

FIGURE 21. Neutral stability curve as a function of the overall friction parameter, $S$, and the drag differential, $\gamma$. Note that the curve asymptotes to $S=0$ at $\gamma=0$. Also shown are data from the present experiments $(\triangle)$ (vortex instability observed), and data from the two-array experiments of Lightbody \& Nepf (2006) ( $\square$ ) (no vortex instability observed).

the two-dimensional instability problem for a porous layer adjacent to a frictionless channel. These results suggest that when friction in the channel is negligibly small compared with the resistance in the porous layer, the shear layer will at least be neutrally stable even for very dense arrays. This assumes the layer porosity is always sufficient to achieve the velocity inflection point necessary for instability. 


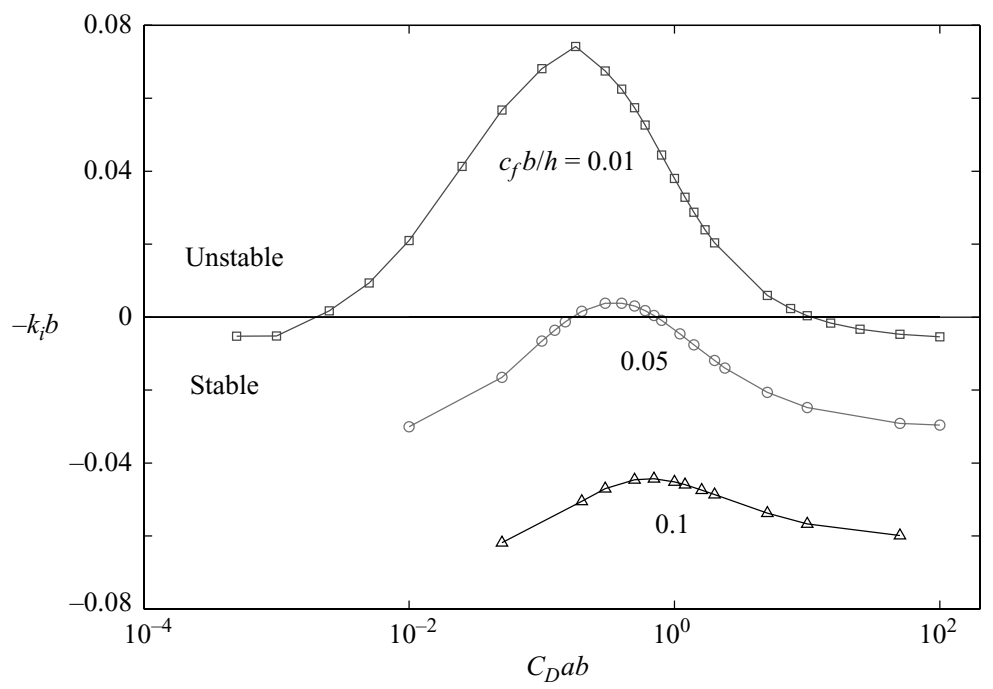

FIGURE 22. Growth rate of the most unstable mode with increasing array density for varying degrees of background bed friction.

All conditions examined in the present experiments lie within the unstable range (figure 21), consistent with our observations of coherent vortices for all cases. In addition, the theory correctly predicts the most unstable frequency, $f \theta / \bar{U}=0.032$. The experimental conditions of Lightbody \& Nepf (2006), in a channel with two adjacent cylinder arrays with different density, are in a stable $(\gamma, S)$ region. Consistent with the theory, they did not observe coherent oscillations at the interface between the arrays. Thus the linear theory correctly predicts the conditions leading to instability. The agreement suggests that the source of the coherent vortices observed in the present experiments is an instability which is both fundamentally two-dimensional and, since a quadratic friction model is sufficient to capture it, is not overly sensitive to the small-scale details of the roughness layer.

From the neutral curve, we can see whether a particular combination of array drag, $C_{D} a b$, and background bed friction, $c_{f} b / h$, will lead to shear instability. If $c_{f} b / h$ is large enough, increasing the array density can have a stabilizing effect, by increasing $S$. On the other hand, if $c_{f} b / h$ is small, increasing the density favours instability by increasing the drag differential, $\gamma$. These competing tendencies are illustrated in figure 22, which shows the change in growth rate as the array drag is increased for fixed values of the background bed friction. Starting with small uniform bed friction, $c_{f} b / h=0.01$, an increase in array density, $C_{D} a b$, makes the shear layer transition from stable to unstable at an intermediate density, and then back to stable at higher density. However, for high bed friction, $c_{f} b / h=0.1$, the flow is always stable regardless of array density. It can be concluded that dense arrays adjacent to a relatively frictionless open region will favour instability owing to the drag differential, a result that is consistent with observations of instability in our experiments. However, for adjacent layers, which each have significant drag, such as two layers of different porosity, instabilities will tend to be damped.

\section{Conclusions}

Experimental results are presented for shear instability in a shallow channel partially filled with a model porous layer of circular cylinders. The drag differential between 
the porous layer and the open region creates an inflection point in the mean velocity, leading to an instability characterized by regular coherent vortices. The vortices reach an equilibrium with regular oscillations at a frequency predicted by linear stability theory. The equilibrium appears to be maintained by a cycle consisting of sweeps which carry high-momentum fluid toward the interface, enhancing shear, and leading to energetic ejections that transfer momentum and reinforce the sweeps. The momentum transfers lead to a friction coefficient that is an order of magnitude larger than for a solid wall. The coherent structures are also expected to increase mass transport substantially near a roughness layer, and thus have potential relevance to exchange across, for example, fringing aquatic vegetation, marine benthic boundary layers, and river hyporheic zones.

The shear layer is found to possess two distinct length scales. The inner-layer thickness is set by the array resistance. The wider outer region, which resembles a boundary layer, has a width set by the water depth and bottom friction. The instability is established by the inner layer, near the velocity inflection point, but the size of the resulting coherent vortices and their passage frequency are set by the outerlayer characteristics. The outer-layer scaling implies that, for these experiments, the details of the porous layer are not important in establishing the vortex structure. This conclusion can only be made for the cylinder array studied here; results are required from a wider range of porous-layer geometries and densities before a generalization can be confirmed.

A linear stability analysis demonstrates that while the drag differential maintained by the porous layer reinforces the shear instability, the overall drag may damp it if the background friction in the channel is too great, thus allowing a range of stability characteristics. The analysis also suggests that the instability is fundamentally two-dimensional, and requires only a penetrable roughness layer to be initiated. This conclusion is consistent with the vortex cycle hypothesized to describe the present observations, and also with previous observations of coherent oscillations in a wide range of canopy flows, rough boundary layers, and two-dimensional numerical calculations near roughness layers. It appears that oscillations driven by a shear instability are characteristic to many flows near porous layers.

This material is based upon work supported by the National Science Foundation under Grant 0125056 . Any opinions, findings, and conclusions or recommendations expressed in this material are those of the authors and do not necessarily reflect the views of the National Science Foundation.

\section{REFERENCES}

Beavers, G. \& JosePh, D. 1967 Boundary conditions at a naturally permeable wall. J. Fluid Mech. 30, 197-207.

Bonnet, J., Delville, J., Glauser, M., Antnonia, R., Bisset, D., Cole, D., Fiedler, H., Garem, J., Hillberg, D., Jeong, J., Kevlahan, N., Ukeiley, L. \& Vincendeau, E. 1998 Collaborative testing of eddy structure identification methods in free turbulent shear flows. Exps. Fluids 25, $197-225$.

Bousmar, D., Riviere, N., Proust, S., Paquier, A., Morel, R. \& Zech, Y. 2005 Upstream discharge distribution in compound-channel flumes. J. Hydraul. Engng ASCE 131, 408-412.

Breugem, W., Boersma, B. \& Uittenbogand, R. 2006 The influence of wall permeability on turbulent channel flow. J. Fluid Mech. 562, 35-72.

Browand, F. \& Weidman, P. 1976 Large scales in the developing mixing layer. J. Fluid Mech. 76, 127. 
Brown, G. \& Roshko, A. 1974 On density effects and large structure in turbulent mixing layers. J. Fluid Mech. 64, 775-816.

Chen, D. \& JiRKA, G. 1997 Absolute and convective instabilities of plane turbulent wakes in a shallow water layer. J. Fluid Mech. 338, 157-172.

Chu, V., Wu, J.-H. \& Khayat, R. 1991 Stability of transverse shear flows in shallow open channels. J. Hydraul. Engng ASCE 117, 1370-1388.

Djenidi, L., Anselmet, F. \& Antonia, R. 1994 LDA measurements in a turbulent boundary layer over a $d$-type rough wall. Exps. Fluids 16, 323-329.

Djenidi, L., Elavarasan, R. \& Antonia, R. 1999 The turbulent boundary layer over transverse square cavities. J. Fluid Mech. 395, 271-294.

Drazin, P. \& ReID, W. 1981 Hydrodynamic Stability. Cambridge University Press.

Finnigan, J. 2000 Turbulence in plant canopies. Annu. Rev. Fluid Mech. 32, 519-571.

Fu, H. \& Rockwell, D. 2005 Shallow flow past a cylinder: control of the near wake. J. Fluid Mech. 539, $1-24$.

Ghaddar, N., Korczak, K., Mikic, B. \& Patera, A. $1986 a$ Numerical investigation of incompressible flow in grooved channels. Part 1. Stability and self-sustained oscillations. J. Fluid Mech. 163, 99-127.

Ghaddar, N. K., Magen, M., Mikic, B. B. \& Patera, A. T. $1986 b$ Numerical investigation of incompressible flow in grooved channels. Part 2. Resonance and oscillatory heat-transfer enhancement. J. Fluid Mech. 168, 541-567.

Ghidaoui, M. \& Kolyshinin, A. 1999 Linear stability analysis of lateral motions in compound open channels. J. Hydraul. Engng ASCE 125, 871-880.

GhisalberTi, M. \& NePF, H. M. 2002 Mixing layers and coherent structures in vegetated aquatic flows. J. Geophys. Res. Oceans 107 (C2), 3011.

Ho, C.-M. \& Huerre, P. 1984 Perturbed free shear layers. Annu. Rev. Fluid Mech. 16, 365-424.

Hussain, A. 1983 Coherent structures - reality and myth. Phys. Fluids 26 (10), 2816-2850.

JAMES, D. \& Davis, A. 2001 Flow at the interface of a model fibrous porous medium. J. Fluid Mech. 426, 47-72.

Jimenez, J., Uhlmann, M., Pinelli, A. \& Kawahara, G. 2001 Turbulent shear flow over active and passive porous surfaces. J. Fluid Mech. 442, 89-117.

Koch, D. \& LADD, A. 1997 Moderate Reynolds number flows through periodic and random arrays of aligned cylinders. J. Fluid Mech. 349, 31-66.

LeONARD, L. \& Luther, M. 1995 Flow hydrodynamics in tidal marsh canopies. Limnol. Oceanogr. 40, 1474-1484.

Lightbody, A. \& Nepf, H. 2006 Prediction of near-field shear dispersion in an emergent canopy with heterogeneous morphology. Environ. Fluid Mech. 6, 477-488.

Monkewitz, P. \& Huerre, P. 1982 Influence of the velocity ratio on the spatial instability of mixing layers. Phys. Fluids 25, 1137-1143.

NADAOKA, K. \& YagI, H. 1998 Shallow-water turbulence modeling and horizontal large-eddy computation of river flow. J. Hydraul. Engng ASCE 124, 493-500.

Nepf, H., Ghisalberti, M., White, B. \& Murphy, E. 2007 Retention time and dispersion associated with submerged aquatic canopies. Water Resour. Res. 43, W04422, doi:10.1029/ 2006WR005362.

Nepf, H. M. \& Vivoni, E. R. 2000 Flow structure in depth-limited, vegetated flow. J. Geophys. Res. 105 (C12), $28527-28546$.

Nezu, I. \& Onitsuka, K. 2000 Turbulent structures in partly vegetated open-channel flows with LDA and PIV measurements. J. Hydraul. Res. 39, 629-642.

Pedras, M. \& De Lemos, M. 2000 On the definition of turbulent kinetic energy for flow in porous media. Intl Commun. Heat Mass Transfer 27, 211-220.

Perry, A. \& ChOng, M. 1987 A description of eddying motions and flow patterns using critical-point concepts. Annu. Rev. Fluid Mech. 19, 125-155.

Pierrehumbert, R. \& Widnall, S. 1982 The two- and three-dimensional instabilities of a spatially periodic shear layer. J. Fluid Mech. 114, 59-82.

Poggi, D., Porporato, A. \& Ridolfi, L. 2004a The effect of vegetation density on canopy sub-layer turbulence. Boundary-Layer Met. 111, 565-587.

Poggi, D., Katul, G. G. \& Albertson, J. D. 2004b A note on the contribution of dispersive fluxes to momentum transfer within canopies. Boundary-Layer Met. 111, 615-621. 
Pope, S. B. 2000 Turbulent Flows. Cambridge University Press.

van Prooijen, B., Battjes, J. \& Uijttewaal, W. 2005 Momentum exchange in straight uniform compound channel flow. J. Hydraul. Engng ASCE 131, 177-185.

Raupach, M. \& Thом, M. 1981 Turbulence in and above plant canopies. Annu. Rev. Fluid Mech. 13, 97-129.

Raupach, M., Finnigan, J. \& Brunet, Y. 1996 Coherent eddies and turbulence in vegetation canopies: the mixing layer analogy. Boundary-Layer Met. 78, 351-382.

Schatz, M., Barkley, D. \& Swinney, H. 1995 Instability in a spatially periodic open flow. Phys. Fluids 7 (2), 344-358.

Schlichting, H. 1979 Boundary-Layer Theory, 7th edn. McGraw-Hill.

Shvidchenko, A. \& Pender, G. 2001 Macroturbulent structure of open-channel flow over gravel beds. Water Resour. Res. 37, 709-719.

Socolofsky, S. \& JiRka, G. 2004 Large scale flow structures and stability in shallow flows. J. Environ. Engng Sci. 3, 451-462.

Tamai, N., Asaeda, T. \& IKedA, H. 1986 Study on generation of periodical large surface eddies in a composite channel flow. Water Resour. Res. 22, 1129-1138.

UijttewaAl, W. \& Boois, R. 2000 Effects of shallowness on the development of free-surface mixing layers. Phys. Fluids 12, 392-402.

UijtTEWAal, W. \& JiRka, G. 2003 Grid turbulelence in shallow flows. J. Fluid Mech. 489, 325-344.

UiJTtewaAl, W. \& TUKKer, J. 1998 Development of quasi two-dimensional structures in a shallow free-surface mixing layer. Exps. Fluids 24, 192-200.

VReugdenhill, C. 1994 Numerical Methods for Shallow-Water Flow. Kluwer.

Winant, C. \& Browand, F. 1974 Vortex pairing, the mechanism of turbulent mixing-layer growth at moderate Reynolds number. J. Fluid Mech. 63, 237-255.

Xiaohui, S. \& Li, C. 2002 Large eddy simulation of free surface turbulent flow in partly vegetated open channels. Intl J. Numer. Meth. Fluids 39, 919-937.

Zaman, K. \& Hussain, A. 1981 Taylor hypothesis and large-scale coherent structures. J. Fluid Mech. 112, 379-396. 